\title{
Transition to blow-up in a reaction-diffusion model with localized spike solutions $\dagger$
}

\author{
V. ROTTSCHÄFER ${ }^{1}$, J. C. TZOU ${ }^{2}$ and M. J. WARD ${ }^{2}$ \\ ${ }^{1}$ Mathematical Institute, Leiden University, P.O. Box 9512, Leiden, the Netherlands \\ email: vivi@math.leidenuniv.nl \\ ${ }^{2}$ Department of Mathematics, University of British Columbia, Vancouver, British Columbia, V6T 1Z2, Canada \\ emails: tzou.justin@gmail.com, ward@math.ubc.ca
}

(Received 6 September 2016; revised 1 February 2017; accepted 2 February 2017;

first published online 7 March 2017)

\begin{abstract}
For certain singularly perturbed two-component reaction-diffusion systems, the bifurcation diagram of steady-state spike solutions is characterized by a saddle-node behaviour in terms of some parameter in the system. For some such systems, such as the Gray-Scott model, a spike self-replication behaviour is observed as the parameter varies across the saddle-node point. We demonstrate and analyse a qualitatively new type of transition as a parameter is slowly decreased below the saddle node value, which is characterized by a finite-time blow-up of the spike solution. More specifically, we use a blend of asymptotic analysis, linear stability theory, and full numerical computations to analyse a wide variety of dynamical instabilities, and ultimately finite-time blow-up behaviour, for localized spike solutions that occur as a parameter $\beta$ is slowly ramped in time below various linear stability and existence thresholds associated with steady-state spike solutions. The transition or route to an ultimate finitetime blow-up can include spike nucleation, spike annihilation, or spike amplitude oscillation, depending on the specific parameter regime. Our detailed analysis of the existence and linear stability of multi-spike patterns, through the analysis of an explicitly solvable nonlocal eigenvalue problem, provides a theoretical guide for predicting which transition will be realized. Finally, we analyse the blow-up profile for a shadow limit of the reaction-diffusion system. For the resulting non-local scalar parabolic problem, we derive an explicit expression for the blow-up rate near the parameter range where blow-up is predicted. This blow-up rate is confirmed with full numerical simulations of the full PDE. Moreover, we analyse the linear stability of this solution that blows up in finite time.
\end{abstract}

Key words: Nonlocal eigenvalue problem, delayed bifurcation, saddle node, Hopf bifurcation, finite-time blow-up.

\section{Introduction}

In the singularly perturbed limit of small diffusivity, certain two-component reactiondiffusion (RD) systems in a one-dimensional spatial domain can support the existence of localized spike-type solutions, whereby one of the two components becomes localized around certain points in the domain. A prototypical system admitting spike patterns is the

$\dagger$ M. J. Ward was supported by an NSERC Discovery Grant 81541 (Canada). J. C. Tzou was supported by a PIMS postdoctoral fellowship. 
Gierer-Meinhardt (GM) model (cf. [14]). Nowadays, there exists a rather well-developed theory for the existence, linear stability, and dynamics of localized spike patterns for many two-component RD systems in one dimension (see [5-7, 15, 16, 35] for some of the earlier studies). A much more extensive bibliography is given in [25].

For some of the RD systems, the bifurcation diagram of steady-state spike solutions is characterized by a saddle-node behaviour in terms of some parameter in the system. In particular, for the well-known Gray-Scott RD system transitions in a feed-rate parameter past some critical saddle-node bifurcation point are known to lead to spike self-replication behaviour, whereby a spike dynamically splits into two. Further transitions in the feedrate parameter across other saddle-node points of multi-spike equilibria lead to the subsequent generation of additional spikes through a dynamical splitting phenomenon (cf. [5, 8, 18, 19, 27]).

The goal of this paper is to analyse spike solutions for a RD model that exhibits a qualitatively new type of transition as a bifurcation parameter crosses a saddle-node value. More specifically, we will analyse the linear stability and transition to finite-time blow-up behaviour of localized spike solutions to the RD system:

$$
\begin{aligned}
& v_{t}=\varepsilon^{2} v_{x x}+v-v u+v^{3}, \quad|x| \leqslant 1, \quad t>0 ; \quad v_{x}( \pm 1, t)=0, \\
& \tau u_{t}=D u_{x x}-u+2 \varepsilon^{-1} \beta v^{3}, \quad|x| \leqslant 1, \quad t>0 ; \quad u_{x}( \pm 1, t)=0 \text {. }
\end{aligned}
$$

In (1.1), $0<\varepsilon \ll 1, D>0, \beta>0$, and $\tau \geqslant 0$, are constants, with $D=O(1)$ as $\varepsilon \rightarrow 0$. A very similar system, where $\beta v^{3}$ is replaced by $\beta v^{2}$ in $(1.1 b)$, was considered briefly in Section 5 of [19] where blow-up behaviour of localized spike solutions was conjectured. In [7], a related system, representing a variant of the GM model, was also conjectured, based on numerical evidence, to lead to a finite-time blow-up of the spike amplitude.

In the shadow limit where $D \gg 1$ and $\tau=\mathcal{O}(1)$, (1.1) reduces to the following PDEODE model for $v(x, t)$ and $u(t)$ :

$$
\begin{aligned}
v_{t} & =\varepsilon^{2} v_{x x}-(u-1) v+v^{3}, \quad|x| \leqslant 1, \quad t>0 ; \quad v_{x}( \pm 1, t)=0, \\
\tau \frac{d u}{d t} & =-u+\varepsilon^{-1} \beta \int_{-1}^{1} v^{3} d x .
\end{aligned}
$$

In addition, if we assume that $\tau=0$, then (1.2) reduces to the non-local Ginzburg-Landau (GL) model:

$$
v_{t}=\varepsilon^{2} v_{x x}-\left(\beta \varepsilon^{-1} \int_{-1}^{1} v^{3} d x-1\right) v+v^{3}, \quad|x| \leqslant 1, \quad t>0 ; \quad v_{x}( \pm 1, t)=0 .
$$

A minor variant of (1.3), in which the non-local term has $v^{2}$ rather than $v^{3}$ in its integrand, arises in asymptotic theories of certain convection processes (cf. [23], [28]), and was studied in the context of localized steady-state spike solutions in [26]. The extension of the analysis of [26] to a non-local quintic GL model is given in [36].

For (1.1)-(1.3), we will use a combination of asymptotic analysis, linear stability theory, and full numerical computations to investigate a wide variety of dynamical instabilities, and ultimately finite-time blow-up behaviour, for localized spike solutions that occur as $\beta$ 
is slowly ramped in time below various linear stability and existence thresholds associated with steady-state spike solutions. Through full numerical simulations of system (1.1), we will show that when $\beta$ is slowly decreased, a dynamically intricate route, or transition, of either spike nucleation, spike annihilation, or spike amplitude oscillation appears that occurs before a spike ultimately undergoes a finite-time blow-up of its amplitude (see Experiments 1-4 in Section 4.3). The precise transition to blow-up that is observed depends on the parameter regime of $D$ and $\tau$. Our detailed analysis of the existence and linear stability of multi-spike patterns will provide a theoretical guide for predicting which transition will be realized.

One common challenge in the analysis of the linear stability of multi-spike patterns for RD systems is that one must derive precise stability thresholds from the analysis of a non-local eigenvalue problem (NLEP). One key feature of our model (1.1) is that the underlying NLEP problem is "explicitly solvable" in the sense of [25], in that the problem of detecting any unstable eigenvalues of the NLEP is reduced to the highly tractable analytical problem of determining the roots to some explicit transcendental equations in the eigenvalue parameter. A related key feature of (1.1) is that we are also able to provide a detailed analysis of delayed bifurcation behaviour that occurs as $\beta$ is slowly ramped below linear stability thresholds obtained from the NLEP analysis. Our analysis theoretically predicts the delayed value of $\beta$ for which either a competition instability in the spike amplitude occurs for (1.1) or an oscillatory instability in the spike amplitude occurs for a one-spike solution of (1.2).

Delayed bifurcation phenomena have been analysed rather extensively in the context of ODEs. For some early studies of this type, see [1,9] and [22], and for a more recent overview, see [21]. However, in the context of PDEs, there have been relatively few detailed analytical studies of delayed bifurcation. One such study is [33] where a related explicitly solvable NLEP was key to analysing delayed bifurcation behaviour of spike solutions to a specific form of the GM model and a generalization of the Gray-Scott model.

The outline of this paper is as follows.

In Section 2, the method of matched asymptotic expansions is used to construct $k$-spike steady-state solutions to $(1.1)$ in the limit $\varepsilon \rightarrow 0$. This analysis shows that there are critical existence thresholds $\beta_{k}$, with $\beta_{k+1}<\beta_{k}$, for which there exists a $k$-spike steady-state for $\beta>\beta_{k}$ and when $\beta<\beta_{k}$ it does not. This critical value of $\beta$ corresponds to a saddle-node bifurcation point of spike equilibria.

In Section 3, we perform a linear stability analysis of these spike solutions. A novel feature in this analysis, in comparison with similar studies for other models (cf. [5], [35]), is that we must consider both the stability of the background state, defined away from the spikes, as well as the stability of the spike profile. In Section 3, we show that the background state is linearly stable only if $D>D_{\mathrm{b}}$, where $D_{\mathrm{b}}$ depends on $\beta$ and the number of spikes. To analyse the linear stability of the spike profile, we first derive an NLEP in Section 3 that characterizes any instabilities of the amplitudes of the spikes on an $\mathcal{O}(1)$ time-scale. We find that the NLEP is explicitly solvable, and hence, can be readily analysed. This is a consequence of the choice of the non-linearity, namely cubic, in equation $(1.1 b)$, compared to the system studied in Section 5 of [19]. For $0 \leqslant \tau<\tau_{H k}$ and for $k \geqslant 2$, we show that a $k$-spike equilibrium is linearly stable only when $\beta>\beta_{c k}>\beta_{k}$ for some thresholds $\beta_{c k}$ and $\tau_{H k}$. 
The threshold $\beta_{c k}$, referred to as the competition stability threshold, occurs as a result of a zero-eigenvalue crossing and leads to a sign-fluctuating instability in the spike amplitudes. Moreover, the $k$-spike equilibrium with $k \geqslant 2$ is unstable for all $\tau>0$ in the range $\beta_{k}<\beta<\beta_{c k}$. For $k=1$, we have $\beta_{c 1}=\beta_{1}$, and for $\beta>\beta_{1}$, a one-spike steady-state is linearly stable only in the range $0 \leqslant \tau<\tau_{H}$ and undergoes a Hopf bifurcation at $\tau=\tau_{H}$, which depends on $\beta$ and $D$. Asymptotic approximations for $\tau_{H}$ in the limit $D \gg 1$ and for $D \ll 1$ are derived.

Next, we study in Section 4 two specific scenarios in which $\beta$ is slowly decreased. We provide a detailed analysis of the delayed bifurcation that occurs in these two cases. In Section 4.1, we study delayed bifurcation behaviour for a two-spike steady-state of (1.1) with $\tau=0$ when $\beta$ is slowly and linearly ramped in time below the competition threshold $\beta_{c 2}$. The analysis predicts the existence of a critical value of $\beta$, satisfying $\beta_{2}<\beta<\beta_{c 2}$, at which the delayed competition instability is fully realized. For a fixed $\tau>0$, we analyse in Section 4.2 the delayed Hopf bifurcation that occurs for a one-spike solution to the PDE-ODE model (1.2) when $\beta$ is slowly ramped below the Hopf bifurcation threshold associated with the NLEP. We show that the delayed oscillatory instability is fully realized before $\beta$ reaches the existence threshold $\beta_{1}$. In Section 4.3 , we perform several numerical experiments on the RD system (1.1) using FlexPDE6 [12] to confirm the theoretical predictions of delayed instabilities, and to exhibit several distinct dynamical routes, or transitions, that can occur leading to an ultimate finite-time blow-up of a spike amplitude.

In Section 5, we analyse the numerically observed finite-time blow-up behaviour for a solution to the non-local GL model (1.3) whenever $\beta$ is below the saddle node point $\beta_{1}=1 /(\sqrt{2} \pi)$ associated to the existence of a one-spike steady-state. In the analysis, we use a dynamical rescaling approach, based on a near-similarity group transformation, to construct a solution to (1.3) that has finite-time blow-up at $x=x_{0}$. Our analysis is related to the centre-manifold approach developed in [11] (see also [10] for a related study of quenching) for the scalar model $v_{t}=\Delta v+v^{p}$ for $p>1$ and to the more recent studies of [29] and [4] for finite-time blow-up in a complex GL equation and to [2] for a range of different models. In contrast to the study of [11] of blow-up in the local model, our analysis of the non-local model (1.3) requires the numerical computation of a simple BVP characterizing the blow-up profile. We will show for (1.3) that the blow-up profile exists whenever $\beta<\beta_{1}$. In the limit $\beta \rightarrow \beta_{1}^{-}$, we construct the blow-up profile using asymptotic analysis to predict a precise blow-up rate. We also examine the linear stability of the blow-up profile by following the general methodology of [2].

Our analysis of a single-point blow-up for (1.3), similar to that for scalar quasilinear heat equations, differs from that of existing literature for non-local equations. Problems that have been studied are of the form

$$
u_{t}=\Delta u+f(u)-\int_{\Omega} f(u) d x
$$

with $\partial_{n} u=0$ and a function $f(u)$ that is usually taken to be a power of $u$. For $f(u)=u^{p}$, it was shown in [3] in one dimension that $u$ blows up at each point in $\Omega$, but with a non-uniform blow-up rate depending on the point $x \in \Omega$. Moreover, in [34], an estimate for the blow-up rate of non-global solutions for $f(u)=u^{2}$ was established. 
The case when $f(u)=u|u|^{p-1}$ was studied by [13] and the problem where $f(u)=|u|^{p}$ was considered in $[17,30]$. In both cases, it was shown that for any $p>1$, the solution must blow-up if the initial data satisfies a certain energy condition. Similar global blow-up behaviour was shown for the more general model:

$$
u_{t}=\Delta u-f_{1}(u, \nabla u)+u^{m}\left(\int_{\Omega} u^{k}\right)^{\frac{p}{k}} d x
$$

on $\Omega$ with Dirichlet boundary conditions and under certain conditions on the function $f_{1}$ (for more details, see [31,32]). However, due to the different signs in front of the integral term in comparison to our problem, the theory developed in these articles is not applicable here.

The paper concludes with a brief discussion in Section 6.

\section{Asymptotic construction of steady-state spike solutions}

In this section, we use the method of matched asymptotic expansions for $\varepsilon \ll 0$ to construct a $k$-spike steady-state solution to (1.1), with $k \geqslant 1$ evenly spaced interior spikes, on the interval $|x| \leqslant 1$. To do so, we need only construct an even one-spike solution centred at $x=0$ on the interval $|x| \leqslant l$ and then set $l=1 / k$. By translation invariance and through reflection of this canonical one-spike solution, defined on $|x| \leqslant l=1 / k$, a $k$-spike steady-state solution to (1.1) on $|x| \leqslant 1$ is readily obtained.

We consider (1.1) on $|x| \leqslant l$, and construct a stationary spike solution centred at $x=0$. In the inner region near $x=0$, we introduce the new variables $y, V(y)$ and $U(y)$, by

$$
y=x / \epsilon, \quad V(y)=v(\varepsilon y), \quad U(y)=u(\varepsilon y) .
$$

Upon introducing these variables into the steady-state problem for (1.1), we obtain to leading order that $U \sim U_{0}+o(1)$, for some constant $U_{0}$, and that $V(y)$ is a homoclinic solution to $V^{\prime \prime}-\left(U_{0}-1\right) V+V^{3}=0$ where the prime denotes differentiation with respect to $y$. For a spike-solution to exist on $|x| \leqslant l$, we require that $U_{0}>1$, and on this range of $U_{0}$, we get

$$
V(y)=\left(U_{0}-1\right)^{1 / 2} w\left[\sqrt{U_{0}-1} y\right],
$$

where $w(z)=\sqrt{2} \operatorname{sech} z$ is the unique homoclinic solution to

$w^{\prime \prime}-w+w^{3}=0, \quad-\infty<z<\infty ; \quad w \rightarrow 0 \quad$ as $\quad|z| \rightarrow \infty ; \quad w^{\prime}(0)=0, \quad w(0)>0$.

Next, since $v$ is localized near $x=0$, we can use (2.1) to determine, in the sense of distributions, that

$$
\begin{aligned}
& 2 \beta \varepsilon^{-1} v^{3} \rightarrow 2 \beta\left(\int_{-\infty}^{\infty} V^{3} d y\right) \delta(x)=2 \beta\left(U_{0}-1\right)^{3 / 2} \\
& \quad \times\left(\int_{-\infty}^{\infty}\left(w\left[\sqrt{U_{0}-1} y\right]\right)^{3} d y\right) \delta(x)=2 \sqrt{2} \beta \pi\left(U_{0}-1\right) \delta(x) .
\end{aligned}
$$


Here, we have used $\int_{-\infty}^{\infty}[w(z)]^{3} d z=\sqrt{2} \pi$. In this way, it follows from (1.1b) and (2.3), that the steady-state outer solution for $u$ on $|x| \leqslant l$ is

$$
D u_{x x}-u=-2 \sqrt{2} \beta \pi\left(U_{0}-1\right) \delta(x), \quad|x|<l ; \quad u_{x}( \pm l)=0,
$$

together with the matching condition that $u(0)=U_{0}$.

We represent the solution to (2.4) in terms of the Green's function $G(x)$, satisfying

$$
G_{x x}-\frac{1}{D} G=-\delta(x), \quad|x| \leqslant l ; \quad G_{x}( \pm l)=0,
$$

which is given explicitly by

$$
G(x)=\frac{\cosh \left[\omega_{0}(l-|x|)\right]}{2 \omega_{0} \sinh \left(\omega_{0} l\right)}, \quad \omega_{0} \equiv 1 / \sqrt{D} .
$$

In terms of $G(x)$, the solution to (2.4) is

$$
u(x)=2 \sqrt{2} \beta \pi \omega_{0}^{2}\left(U_{0}-1\right) G(x) .
$$

Upon setting $u(0)=U_{0}$ in (2.6) and letting $l=1 / k$, we obtain for a $k$-spike steady-state solution on $|x| \leqslant 1$ that

$$
\beta=\beta_{k}\left[1+\frac{1}{U_{0}-1}\right], \quad \beta_{k} \equiv \frac{1}{\sqrt{2} \pi \omega_{0}} \tanh \left(\frac{\omega_{0}}{k}\right),
$$

which determines $\beta$ in terms of $U_{0}$. Since we require $U_{0}>1$, we conclude that a $k$-interiorspike steady-state solution exists only when $\beta>\beta_{k}$, where $\beta_{k}$ is the existence threshold for the $k$-spike solution. Moreover, as $U_{0} \rightarrow 1$, we see that $\beta \rightarrow \beta_{k}$. Also, $\beta_{k+1}<\beta_{k}$ and $\beta_{k}$ limits to zero as $k$ increases. The limiting asymptotics of $\beta_{k}$ for both large and small diffusivity is

$$
\beta_{k} \sim \frac{1}{\sqrt{2} \pi k}, \quad D \gg 1 ; \quad \beta_{k} \sim \frac{1}{\pi} \sqrt{\frac{D}{2}}, \quad D \ll 1 .
$$

In Figure 1, we plot for $D=1$, the solution branches consisting of $|v|_{\infty} \equiv w(0) \sqrt{U_{0}-1}$, where $w(0)=\sqrt{2}$, versus $\beta$, as obtained from (2.7), for $k$-spike steady-state solutions to (1.1) for $k=1, \ldots, 4$. In this figure, we also illustrate the stability results obtained in Section 3 from the NLEP associated with the spike profile.

\section{The linear stability analysis}

In this section, we analyse the linear stability of the steady-state spike solutions constructed in Section 2. To do so, we let $v_{e}$ and $u_{e}$ denote the steady-state solution, and we introduce the perturbation

$$
v=v_{e}+e^{\lambda t} \phi, \quad u=u_{e}+e^{\lambda t} \eta
$$




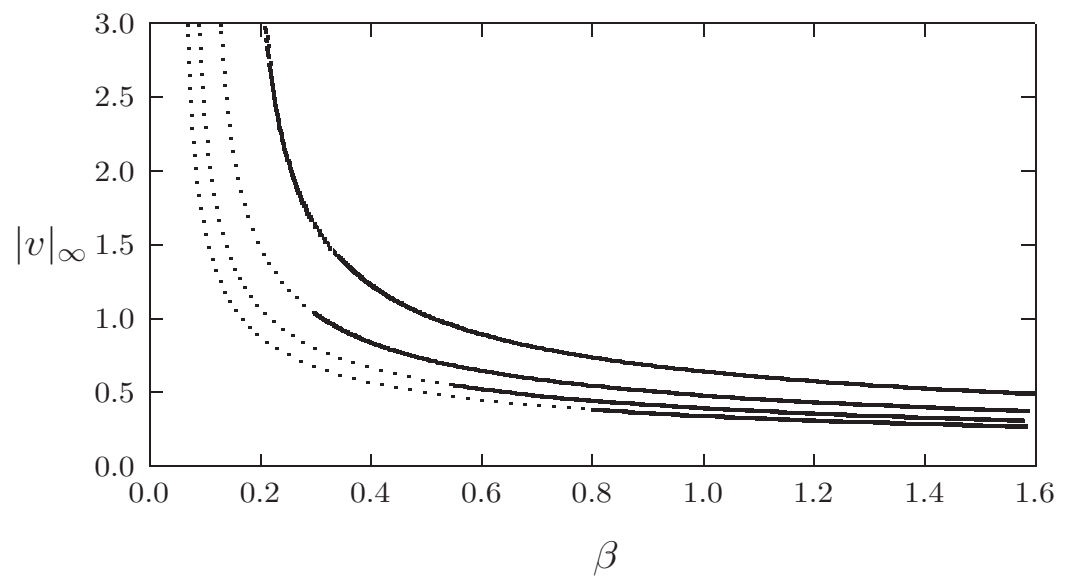

Figure 1. The bifurcation diagram of $|v|_{\infty}=\sqrt{2\left(U_{0}-1\right)}$ versus $\beta$ when $D=1$ for $k$-spike steadystate solutions of $(1.1)$ for $k=1, \ldots, 4$. The heavy solid portions of the curves are where the NLEP associated with the $k$-spike solution has stable eigenvalues when $0 \leqslant \tau<\tau_{H k}$, where $\tau_{H k}$ is a Hopf bifurcation threshold depending on $\beta$ and $D$. The dashed portions indicate where the NLEP has an unstable eigenvalue for any $\tau \geqslant 0$ due to a competition instability. The curves from top to bottom correspond to increasing values of $k$.

Upon substituting (3.1) into (1.1) and linearizing, we obtain that $\phi$ and $\eta$ satisfy

$$
\begin{gathered}
\varepsilon^{2} \phi_{x x}-\left(u_{e}-1\right) \phi+3 v_{e}^{2} \phi-v_{e} \eta=\lambda \phi \\
D \eta_{x x}-(1+\tau \lambda) \eta=-6 \varepsilon^{-1} \beta v_{e}^{2} \phi .
\end{gathered}
$$

For a one-spike solution, we will analyse (3.2) directly subject to $\phi_{x}( \pm 1)=\psi_{x}( \pm 1)=0$. For the multi-spike case, (3.2) will be analysed using a Floquet-based approach, using Floquet boundary conditions on the canonical domain $|x| \leqslant l$ (see Section 3.2 below).

In our linear stability analysis, we must, in addition to deriving an NLEP associated with localized eigenfunctions near the spike locations, also consider the stability of the background outer solution $v_{e}=0$. This is in contrast to similar stability analyses of $k$-spike steady-state solutions for the GM and related models (see [35] and the references therein).

More specifically, from (3.2a), we conclude for $\varepsilon \ll 1$ that the outer solution $v_{e}=0$ is linearly stable for a $k$-spike pattern only when $u_{e}-1>0$ on $\mathcal{O}(\varepsilon)<|x| \leqslant l$, with $l=1 / k$. The outer solution $u_{e}$ is given by expression (2.6) together with $u(0)=U_{0}$ and can hence be expressed as

$$
u_{e}(x)=U_{0} G(x) / G(0)
$$

where $G(x)$ is given in $(2.5 b)$.

Thus, $u_{e}$ decreases monotone on $0<x<l$, and therefore, it follows that $u_{e}(x)>1$ on $0<x<l$ only when $u_{e}(l)>1$. By evaluating the outer solution $u_{e}(x)$ at $x=l=1 / k$, we conclude that the background state is linearly stable if and only if $U_{0} \operatorname{sech}\left(\omega_{0} / k\right)>1$. Upon setting $U_{0} \operatorname{sech}\left(\omega_{0} / k\right)=1$, we obtain a curve in the $D$ versus $\beta$ parameter plane, 


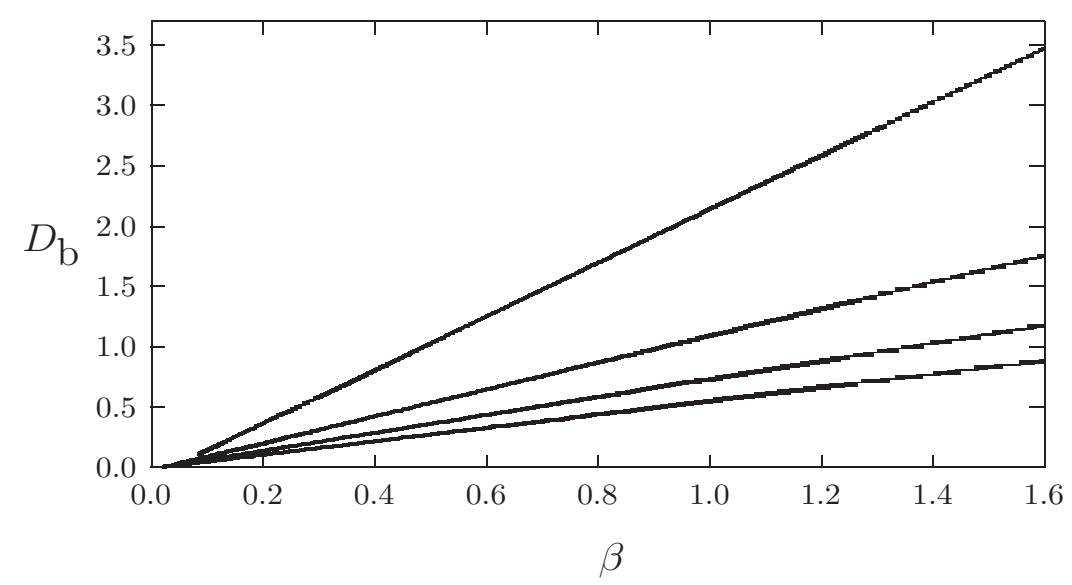

FIGURE 2. The stability threshold $D_{\mathrm{b}}$ versus $\beta$, obtained from (3.3), characterizing the linear stability of the background state $v_{e}=0$ for patterns with $k=1, \ldots, 4$ steady-state spikes. The curves from top to bottom correspond to increasing values of $k$. For each $k$, the background state is linearly stable when $D>D_{\mathrm{b}}$, otherwise it is unstable.

parameterized by $U_{0}>1$, defined by

$$
D \equiv \frac{1}{k^{2}\left(\log \left[U_{0}+\sqrt{U_{0}^{2}-1}\right]\right)^{2}}, \quad \beta=\frac{\sqrt{D}}{\sqrt{2} \pi} \tanh \left(\frac{1}{k \sqrt{D}}\right)\left(1+\frac{1}{U_{0}-1}\right)
$$

From solving $U_{0} \operatorname{sech}\left(\omega_{0} / k\right)=1$, we also obtain the second solution $k \sqrt{D}=$ $\frac{1}{\log \left[U_{0}-\sqrt{U_{0}^{2}-1}\right]}$, however, since the argument of the logarithm of this expression is smaller than 1 , this does not lead to a solution.

Upon eliminating $U_{0}$, we conclude that if $D>D_{\mathrm{b}}$, where $D_{\mathrm{b}}$ is the unique root of the transcendental equation

$$
\beta_{b}=\frac{1}{\pi} \sqrt{\frac{D}{2}} \operatorname{coth}\left(\frac{1}{2 k \sqrt{D}}\right),
$$

then the background state $v_{e}=0$ is linearly stable. Otherwise, the background state is unstable. A plot of the stability boundary $D_{\mathrm{b}}$ versus $\beta$ is shown in Figure 2 for $k=1, \ldots, 4$. Qualitatively, this result shows that only if $D$ is sufficiently large, so that the inhibitor concentration does not become too small at the midpoint between adjacent spikes, will the background state $v_{e}=0$ for the activator be linearly stable.

In Section 3.1, we derive the NLEP associated with a one-spike solution, and we determine parameter ranges for which this NLEP has no unstable eigenvalues. The corresponding problem for multi-spike solutions is examined in Section 3.2.

\subsection{The NLEP problem for a one-spike solution}

In this section, we derive the NLEP associated with the stability of a one-spike solution centred at $x=0$. As such, we consider (3.2) on $|x| \leqslant 1$ with Neumann conditions $\eta_{x}( \pm 1)=\phi_{x}( \pm 1)=0$. 
In the inner region near $x=0$, we have that $u_{e} \sim U_{0}$ and $v_{e}(x) \sim \sqrt{U_{0}-1} w\left(\sqrt{U_{0}-1} \frac{x}{\varepsilon}\right)$ from (2.1) and $w$ is the pulse solution $w(z) \equiv \sqrt{2} \operatorname{sech}(z)$. Therefore, since $v_{e}$ is localized, $\phi$ will be localized near $x=0$, while $\eta=\eta_{0}+o(1)$ for some $\eta_{0}$ to be determined and for $|x| \leqslant \mathcal{O}(\varepsilon)$. More specifically, we look for a localized eigenfunction for $\phi$ of the form:

$$
\phi(x)=\Phi(z), \quad \text { where } z \equiv \sqrt{U_{0}-1}\left(\frac{x}{\varepsilon}\right) .
$$

Upon substituting (3.4) into (3.2a), we obtain to leading-order that $\Phi(z)$ satisfies

$$
L_{0} \Phi-\frac{w(z)}{\sqrt{U_{0}-1}} \eta_{0}=\frac{\lambda}{U_{0}-1} \Phi, \quad-\infty<z<\infty ; \quad \Phi \rightarrow 0, \quad \text { as } \quad|z| \rightarrow \infty .
$$

Here, $L_{0}$ is the local operator, defined in terms of the pulse solution given above, by

$$
L_{0} \Phi \equiv \Phi_{z z}-\Phi+3 w^{2} \Phi
$$

To determine $\eta_{0}$ in $(3.5 a)$, we must first determine the limiting problem for the outer eigenfunction $\eta(x)$ from $(3.2 b)$. Since $v_{e}$ is localized near $x=0$, we calculate in the sense of distributions that

$$
6 \varepsilon^{-1} \beta v_{e}^{2} \phi \rightarrow 6 \beta \sqrt{U_{0}-1}\left(\int_{-\infty}^{\infty} w^{2} \Phi d z\right) \delta(x)
$$

In this way, we obtain that the outer approximation for $\eta$ satisfies

$$
\eta_{x x}-\theta_{\lambda}^{2} \eta=-6 \beta \omega_{0}^{2} \sqrt{U_{0}-1}\left(\int_{-\infty}^{\infty} w^{2} \Phi d z\right) \delta(x), \quad|x| \leqslant 1 ; \quad \eta_{x}( \pm 1)=0,
$$

subject to the matching condition that $\eta(0)=\eta_{0}$. In (3.7), we defined

$$
\theta_{\lambda} \equiv \sqrt{(1+\tau \lambda) / D}
$$

where we have specified the principal value of the square root to ensure that $\eta(0)$ is analytic in $\operatorname{Re}(\lambda) \geqslant 0$.

We represent the solution to (3.7) in terms of the $\lambda$-dependent Green's function $G_{\lambda}(x)$ satisfying

$$
G_{\lambda, x x}-\theta_{\lambda}^{2} G_{\lambda}=-\delta(x), \quad|x| \leqslant 1 ; \quad G_{\lambda, x}( \pm 1)=0,
$$

which has the explicit solution

$$
G_{\lambda}(x)=\frac{\cosh \left[\theta_{\lambda}(1-|x|)\right]}{2 \theta_{\lambda} \sinh \left(\theta_{\lambda}\right)}
$$

In terms of $G_{\lambda}(x)$, the solution to (3.7) is

$$
\eta(x)=6 \beta \omega_{0}^{2} \sqrt{U_{0}-1}\left(\int_{-\infty}^{\infty} w^{2} \Phi d z\right) G_{\lambda}(x) .
$$


We then set $x=0$ in (3.10) and solve for $\eta(0) \equiv \eta_{0}$. Finally, upon substituting the resulting expression for $\eta_{0}$ into (3.5a) and using that $\int_{-\infty}^{\infty} w^{3} d z=\sqrt{2} \pi$, we obtain the following NLEP for $\Phi(z)$ :

Principal Result 3.1 For $0<\varepsilon \ll 1$, the NLEP associated with the stability of a one-spike steady-state solution for (1.1) is

$$
L_{0} \Phi-\chi_{0} w\left(\frac{\int_{-\infty}^{\infty} w^{2} \Phi d z}{\int_{-\infty}^{\infty} w^{3} d z}\right)=\frac{\lambda}{U_{0}-1} \Phi, \quad-\infty<z<\infty ; \quad \Phi \rightarrow 0, \quad \text { as } \quad|z| \rightarrow \infty .
$$

Here $L_{0}$ is defined in (3.5b), and the multiplier $\chi_{0}=\chi_{0}(\tau \lambda)$ of the nonlocal term is

$$
\chi_{0}(\tau \lambda) \equiv \frac{3 \omega_{0} \tanh \omega_{0}}{\theta_{\lambda} \tanh \left(\theta_{\lambda}\right)}\left(1+\frac{1}{U_{0}-1}\right)
$$

where $\omega_{0} \equiv 1 / \sqrt{D}$ and $\theta_{\lambda}$ is defined in (3.8). Here $U_{0}$ is defined in terms of $\beta$ and $D$ by (2.7) with $k=1$.

We now show that the problem of determining unstable discrete eigenvalues of (3.11) in $\operatorname{Re}(\lambda)>0$ can be reduced to the simpler problem of determining the roots to a simple explicit transcendental equation in the eigenvalue parameter.

Lemma 3.1 Any unstable eigenvalue of (3.11) in $\operatorname{Re}(\lambda)>0$ must be a root of $\mathcal{F}_{0}(\lambda)=0$, where

$$
\mathcal{F}_{0}(\lambda) \equiv \chi_{0}(\tau \lambda)+\frac{\lambda}{U_{0}-1}-3
$$

Proof To prove this, we consider only the region $\operatorname{Re}(\lambda)>-\left(U_{0}-1\right)$, where we can guarantee that $|\Phi| \rightarrow 0$ exponentially as $|z| \rightarrow \infty$ since there the background solution is stable. From this analysis of the stability of the background state, in Section 3, we also know that the continuous spectrum for (3.11) is $\lambda<-\left(U_{0}-1\right)<0$, with $\lambda$ real. To establish (3.12), we use Green's identity on $w^{2}$ and $\Phi$, which is written as $\int_{-\infty}^{\infty}\left(w^{2} L_{0} \Phi-\Phi L_{0} w^{2}\right) d y=0$. Upon using (3.11a), together with the key identity that $L_{0} w^{2}=3 w^{2}$ (see Lemma 2.3 of [25]), the expression above from Green's identity reduces to

$$
\int_{-\infty}^{\infty} w^{2} \Phi d z\left(\chi_{0}+\frac{\lambda}{U_{0}-1}-3\right)=0 .
$$

Therefore, for eigenfunctions for which $\int_{-\infty}^{\infty} w^{2} \Phi d z \neq 0$, (3.12) readily follows. In contrast, for eigenfunctions for which $\int_{-\infty}^{\infty} w^{2} \Phi d z=0$, equation (3.11) reduces to the local eigenvalue problem $L_{0} \Phi=v \Phi$, where $v \equiv \lambda /\left(U_{0}-1\right)$. It was proved in Proposition 5.6 of [6] that the point spectrum of this local eigenvalue problem consists only of $v_{0}=3$, together with translation mode $v_{1}=0$, associated with the odd eigenfunction $\Phi_{1}=w^{\prime}$. For $v_{0}=3$, the eigenfunction satisfies $\Phi_{0}>0$ and consequently $\int_{-\infty}^{\infty} w^{2} \Phi d z \neq 0$. As such, any unstable eigenvalue of the NLEP (3.11) must be a root of $\mathcal{F}_{0}(\lambda)=0$. 
Remark 3.1 Our modification of the non-linearity in (1.1b) to $2 \varepsilon^{-1} \beta v^{3}$ rather than $2 \varepsilon^{-1} \beta v^{2}$, as considered previously in [19], has resulted in an NLEP for which the problem of determining the unstable spectrum is reduced to the simpler problem of determining the roots to an explicit transcendental equation. Further examples of non-linear kinetics that lead to such "explicitly solvable" NLEP problems are discussed in [24] and [25].

We now analyse the roots of $\mathcal{F}_{0}(\lambda)=0$ in $\operatorname{Re}(\lambda) \geqslant 0$. Upon substituting $(3.11 b)$ for $\chi_{0}$ into (3.12), we conclude that any unstable eigenvalue of the NLEP (3.11) is a root of

$$
2 \sqrt{1+\tau \lambda}\left(\frac{\tanh \theta_{\lambda}}{\tanh \omega_{0}}\right)=-\frac{d_{1}}{b-\lambda} \equiv \mathcal{G}(\lambda)
$$

where $\omega_{0}=1 / \sqrt{D}$. Here, $d_{1}$ and $b$ are defined by

$$
d_{1} \equiv-6 U_{0}, \quad b \equiv 3\left(U_{0}-1\right)
$$

Viewing $\tau$ as the bifurcation parameter, we claim that (3.13) can have no zero eigenvalue crossings as $\tau \geqslant 0$ is increased and that there is no positive real eigenvalue when $\tau=0$. On the positive real $\lambda$ axis, this follows since the left-hand side of (3.13) is 2 at $\lambda=0$ for all $\tau \geqslant 0$, that $\mathcal{G}(0)>2$ (as a result of $U_{0}>1$ ), and that $\mathcal{G}(\lambda)$ has a vertical asymptote at $\lambda=3\left(U_{0}-1\right)>0$, and is monotonically increasing and convex on $0<\lambda<3\left(U_{0}-1\right)$. Alternatively, on $\lambda>3\left(U_{0}-1\right)$, we have $\mathcal{G}(\lambda)<0$. Therefore, for $\tau=0$, there is no positive real eigenvalue, and no zero eigenvalue crossings can occur for any $\tau \geqslant 0$. However, for $\tau \gg 1$, it is readily seen that there are exactly two positive roots to (3.13) on $0<\lambda<3\left(U_{0}-1\right)$. By continuity of the eigenvalue paths with respect to $\tau$, this suggests that there should be a Hopf bifurcation as $\tau$ is increased. This discussion can be made rigorous by using a winding number approach, very similar to that done in [25], establishing that (3.13) has a Hopf bifurcation at some critical value of $\tau$, depending on $\beta$ and $D$.

For $\beta>\beta_{1}$, in Figure 3, we show for $D=0.2,1,10$ numerical results for the unique value $\tau=\tau_{H}>0$ for which (3.13) admits a purely complex conjugate pair of eigenvalues $\lambda= \pm i \lambda_{H}$, with $\lambda_{H}>0$. We have $\operatorname{Re}(\lambda)>0$ when $\tau>\tau_{H}$, and $\operatorname{Re}(\lambda)<0$ when $0<\tau<\tau_{H}$. In the limit $D \gg 1$ or $D \ll 1$, we now show that the Hopf bifurcation value $\tau_{H}$ can be calculated analytically.

For $D \gg 1$, for which $\omega_{0} \ll 1,(3.13)$ reduces to

$$
2(1+\tau \lambda)=-\frac{d_{1}}{b-\lambda} \equiv \mathcal{G}(\lambda)
$$

Again, this problem was studied in [25]. Since $d_{1}<0$ and $\mathcal{G}(0)>2$ for any $U_{0}>1$, Principal Result 2.5 of [25] applies and we obtain for $D \gg 1$ and $\beta>\beta_{1}$ that

$$
\tau_{H} \sim \frac{1}{3}\left(\frac{\beta}{\beta_{1}}-1\right), \quad \lambda_{H} \sim 3\left(\frac{\beta}{\beta_{1}}-1\right)^{-1 / 2}, \quad \text { where } \quad \beta_{1} \equiv \frac{1}{\sqrt{2} \pi} .
$$



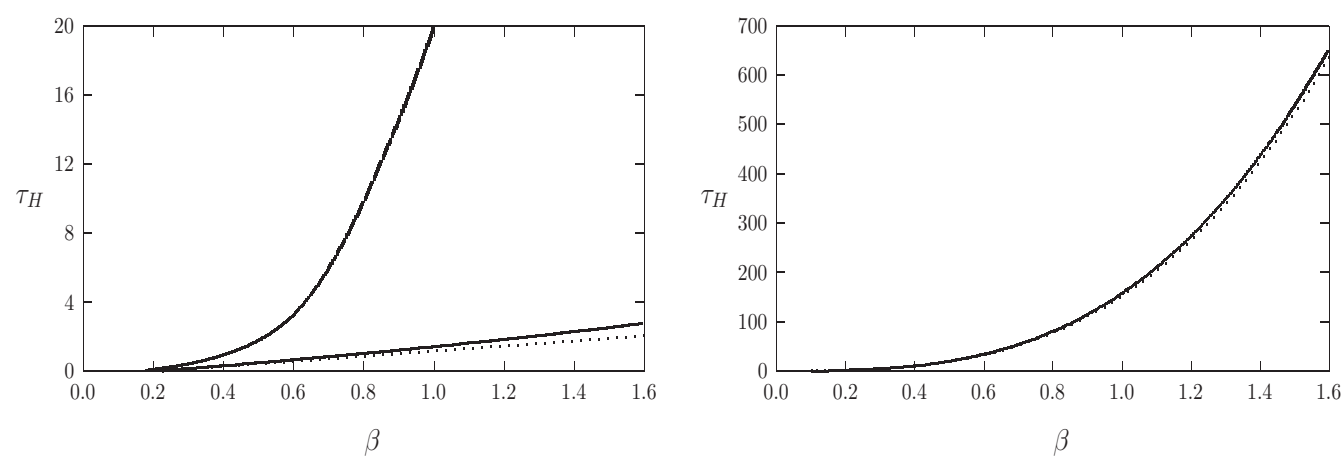

Figure 3. Hopf bifurcation threshold $\tau_{H}$ versus $\beta$ for a one-spike solution. Left panel: $\tau_{H}$ for $D=1$ (top solid curve) and for $D=10$ (bottom solid curve), as computed from (3.13). The dashed curve is the approximation (3.15) for $D$ large. Right panel: $\tau_{H}$ for $D=0.2$ (solid curve), computed from (3.13), compared with the approximation (3.17) for $D$ small (dashed curve).

Alternatively, for $D \ll 1$, for which $\omega_{0} \gg 1$, (3.13) reduces to the following problem:

$$
2 \sqrt{1+\tau \lambda}=-\frac{d_{1}}{b-\lambda} \equiv \mathcal{G}(\lambda)
$$

which was also studied in [25]. From Principal Result 3.8 of [25], we conclude for $D \ll 1$ and $\beta>\beta_{1} \sim \pi^{-1} \sqrt{D / 2}$ that

$$
\begin{aligned}
\tau_{H} & \sim \frac{1}{3}\left(\frac{\beta}{\beta_{1}}-1\right)\left[1+\frac{\beta^{2}}{4 \beta_{1}^{2}}+\frac{\beta}{\sqrt{2} \beta_{1}} \sqrt{1+\frac{\beta^{2}}{8 \beta_{1}^{2}}}\right], \\
\lambda_{H} & \sim 3\left(\frac{\beta}{\beta_{1}}-1\right) \\
-1 & \sqrt{1-\frac{2}{3 \tau_{H}}\left(\frac{\beta}{\beta_{1}}-1\right)} .
\end{aligned}
$$

The limiting expressions for $\tau_{H}$ in (3.17) for small $D$ and for large $D$ in (3.15) are shown in Figure 3 to be rather accurate when $D=10$ and $D=0.2$, respectively, when compared with the Hopf threshold computed from (3.13).

In Figure 4, we re-parametrize the Hopf bifurcation curve by plotting the Hopf bifurcation threshold value $\beta_{H}$, versus $D$ for various values of $\tau>0$. Here, $\beta_{H}$ is the threshold for which the stationary 1-spike solution is linearly stable (unstable) for $\beta_{H}<\beta$ (for $\beta_{H}>\beta$ ). This plot shows that $\beta_{H}>\beta_{1}$ for $\tau>0$, where $\beta_{1}$ is the threshold below which the stationary 1 -spikes do not exist. Therefore, as $\beta$ is adiabatically decreased starting from a value above $\beta_{H}$, a Hopf bifurcation of the spike solution will occur before we reach the steady-state spike-existence threshold $\beta_{1}$.

\subsection{Linear stability of multi-spike solutions}

To analyse the linear stability of the multi-spike steady-state solution $v_{e}$ and $u_{e}$, we will use a Floquet approach in the same spirit as done in [20]. On the interval $|x| \leqslant l$, we 


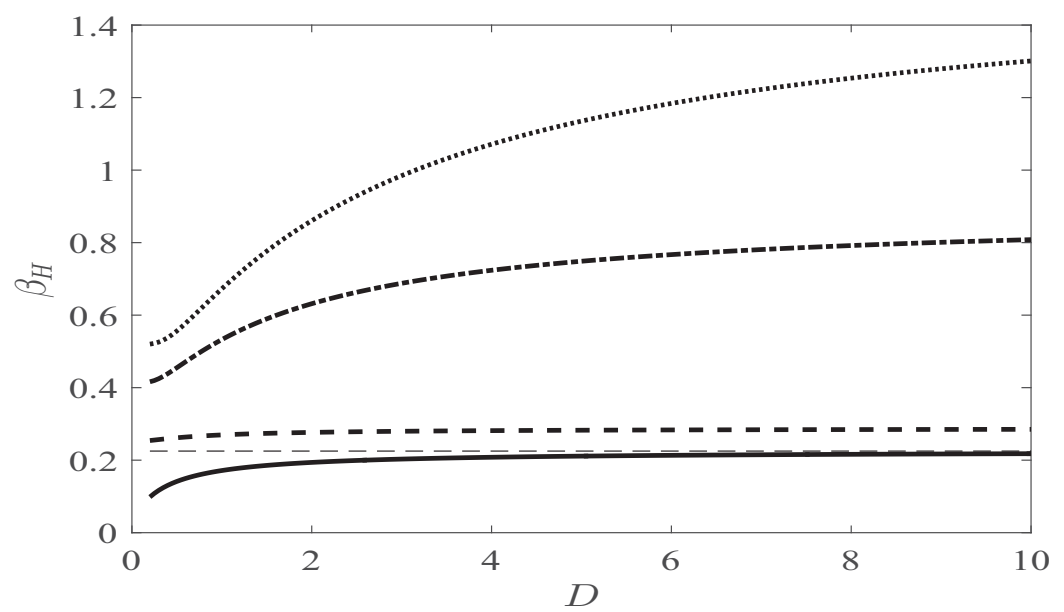

Figure 4. Hopf bifurcation thresholds $\beta_{H}$ versus $D$ for various $\tau$. The one-spike steady-state is linearly stable (unstable) when $\beta<\beta_{H}\left(\beta>\beta_{H}\right)$. The values of $\tau$ are as follows: heavy dashed ( $\tau=0.1)$, dashed-dotted $(\tau=1)$, dotted $(\tau=2)$, hence, $\tau$ increases when going from the lower dashed to the upper curve. The solid curve is the existence threshold $\beta_{1}=\beta_{1}(D)$, while the light dashed line indicates the limiting value $\beta_{1} \rightarrow 1 /(\sqrt{2} \pi)$ as $D \rightarrow \infty$. For any $\tau>0$, the Hopf threshold always occurs before the existence threshold as $\beta$ is decreased.

begin by introducing the perturbation (3.1) into (1.1), to obtain the linearized problem

$$
\begin{gathered}
\varepsilon^{2} \phi_{x x}-\left(u_{e}-1\right) \phi+3 v_{e}^{2} \phi-v_{e} \eta=\lambda \phi, \\
D \eta_{x x}-(1+\tau \lambda) \eta=-6 \varepsilon^{-1} \beta v_{e}^{2} \phi, \quad|x| \leqslant l, \leqslant l,
\end{gathered}
$$

where, in terms of a complex parameter $\xi$, we impose the Floquet-type boundary conditions

$$
\phi(l)=\xi \phi(-l), \quad \phi_{x}(l)=\xi \phi_{x}(-l), \quad \eta(l)=\xi \eta(-l), \quad \eta_{x}(l)=\xi \eta_{x}(-l) .
$$

After deriving the NLEP associated with solving (3.18) for arbitrary $\xi$, we must determine $\xi$ so that our NLEP problem applies to a $K$-spike pattern on the domain $[-l,(2 K-1) l]$ with periodic boundary conditions. This is done by translating $\phi$ and $\psi$ from $[-l, l]$ to the extended interval $[-l,(2 K-1) l]$ in such a way that the extended $\phi$ and $\psi$ have continuous derivatives at $x=l, 3 l, \ldots,(2 K-3) l$. Therefore, since $\phi[(2 K-1) l]=\xi^{K} \phi(-l)$, it follows that to obtain periodic boundary conditions on an interval of length $2 \mathrm{Kl}$, we require that $\xi^{K}=1$, so that

$$
\xi_{j}=e^{2 \pi i j / K}, \quad j=0, \ldots, K-1 .
$$

Upon using these values of $\xi_{j}$ in the NLEP associated with (3.18), we obtain the stability threshold of a $K$-spike steady-state solution on a domain of length $2 K l$ subject to periodic boundary conditions. The last step in the analysis is to extract the stability thresholds for the corresponding Neumann problem from the thresholds for the periodic problem, and 
to choose $l$ appropriately so that the Neumann problem is posed on $[-1,1]$. This is done following (3.26) below.

We now derive the NLEP associated with (3.18). In the inner region near $x=0$, we look as before for a localized inner eigenfunction for $(3.18 a)$ of the form

$$
\phi \sim \Phi(z), \quad \eta \sim \eta_{0}, \quad \text { where } \quad z=\varepsilon^{-1} \sqrt{U_{0}-1} x,
$$

and where $\eta_{0}$ is a constant that is to be determined. Upon using that to leading order $v_{e}^{2} \sim\left(U_{0}-1\right) w^{2}$, we obtain that $\Phi(z)$ satisfies to leading order

$$
L_{0} \Phi-\frac{w}{\sqrt{U_{0}-1}} \eta_{0}=\frac{\lambda}{U_{0}-1} \Phi, \quad-\infty<z<\infty,
$$

where $L_{0}$ is defined in $(3.5 b)$.

To determine the outer approximation for $\eta$ from equation $(3.18 b)$, we use (3.6) to obtain, similar to (3.7), that the outer approximation for $\eta$ satisfies

$$
\begin{aligned}
\eta_{x x}-\theta_{\lambda}^{2} \eta & =-6 \beta \omega_{0}^{2} \sqrt{U_{0}-1}\left(\int_{-\infty}^{\infty} w^{2} \Phi d z\right) \delta(x), \quad|x| \leqslant l ; \quad \eta(l)=\xi \eta(-l), \\
\eta_{x}(l) & =\xi \eta_{x}(-l),
\end{aligned}
$$

where $\theta_{\lambda}$ is defined in (3.8). Again this equation is subject to the matching condition $\eta(0)=\eta_{0}$. We represent the solution to (3.21) in terms of the quasi-periodic Green's function $G_{q}(x)$ satisfying

$$
G_{q, x x}-\theta_{\lambda}^{2} G_{q}=-\delta(x), \quad|x| \leqslant l ; \quad G_{q}(l)=\xi G_{q}(-l), \quad G_{q, x}(l)=\xi G_{q, x}(-l),
$$

to obtain that

$$
\eta(x)=6 \beta \omega_{0}^{2} \sqrt{U_{0}-1}\left(\int_{-\infty}^{\infty} w^{2} \Phi d z\right) G_{q}(x) .
$$

We then evaluate $(3.22 b)$ at $x=0$ to determine $\eta_{0} \equiv \eta(0)$, which is used in (3.20).

To obtain $\eta_{0}$, we must determine $G_{q}(0)$. To do so, we solve $(3.22 a)$ on $-l<x<0$ and on $0<x<l$, and impose the Floquet boundary conditions to obtain

$$
G_{q}(x)= \begin{cases}A \cosh \left(\theta_{\lambda}(x+l)\right)+B \sinh \left(\theta_{\lambda}(x+l)\right), & -l<x<0 \\ \xi A \cosh \left(\theta_{\lambda}(x-l)\right)+\xi B \sinh \left(\theta_{\lambda}(x-l)\right), & 0<x<l\end{cases}
$$

Upon imposing $G_{q}\left(0^{+}\right)=G_{q}\left(0^{-}\right)$and the standard jump condition $G_{q x}\left(0^{+}\right)-G_{q x}\left(0^{-}\right)=$ -1 , as obtained by integrating the ODE in (3.22a) from $x=0^{-}$to $x=0^{+}$, we obtain the linear system for $A$ and $B$ given by

$$
\left(\begin{array}{cc}
(1-\xi) \cosh \left(\theta_{\lambda} l\right), & (1+\xi) \sinh \left(\theta_{\lambda} l\right) \\
\theta_{\lambda}(1+\xi) \sinh \left(\theta_{\lambda} l\right), \theta_{\lambda}(1-\xi) \cosh \left(\theta_{\lambda} l\right)
\end{array}\right)\left(\begin{array}{l}
A \\
B
\end{array}\right)=\left(\begin{array}{l}
0 \\
1
\end{array}\right) .
$$

We then solve for $A$ and $B$ from (3.24), to calculate $G_{q}(0)$ as

$$
G_{q}(0)=A \cosh \left(\theta_{\lambda} l\right)+B \sinh \left(\theta_{\lambda} l\right)=\frac{1}{\theta_{\lambda}}\left[\frac{\cosh \left(\theta_{\lambda} l\right) \sinh \left(\theta_{\lambda} l\right)}{\cosh ^{2}\left(\theta_{\lambda} l\right)+\sinh ^{2}\left(\theta_{\lambda} l\right)-\frac{1}{2}\left(\xi+\xi^{-1}\right)}\right] .
$$


Next, we set $\xi=\xi_{j}=e^{2 \pi i j / K}$ in (3.25), and upon re-arranging the resulting expression, we obtain that

$$
G_{q}(0)=\frac{1}{2 \theta_{\lambda}}\left[\tanh \left(\theta_{\lambda} l\right)+\frac{(1-\cos (2 \pi j / K))}{\sinh \left(2 \theta_{\lambda} l\right)}\right]^{-1} .
$$

Finally, this expression can be used to obtain $\eta_{0}$ from (3.22b). Substituting that into (3.20) results in the NLEP corresponding to a $K$-spike steady-state solution on a domain of length $2 K l$ subject to periodic boundary conditions.

The final step in the stability analysis is to extract the NLEP for the Neumann problem from this NLEP for the periodic problem. More specifically, the stability thresholds for a $k$-spike solution with Neumann boundary conditions can be obtained from the thresholds for a $2 k$-spike solution with periodic boundary conditions on a domain of twice the length. To see this, suppose that $\phi$ is a Neumann eigenfunction on the interval $[0, a]$. If we extend it by an even reflection about the origin to the interval $[-a, a]$, then such an extension satisfies periodic boundary conditions on $[-a, a]$. Alternatively, if $\phi(x)$ is an eigenfunction with periodic boundary conditions at the edge of the interval $[-a, a]$, then if we define $\hat{\phi}(x)=\phi(x)+\phi(-x)$, it follows that $\hat{\phi}$ is an eigenfunction for the Neumann problem on $[0, a]$.

Therefore, to obtain the NLEP governing the stability of a steady-state $k$-spike pattern on an interval of length 2 subject to Neumann boundary conditions, we simply replace $2 K$ by $k$, and hence, $\cos (2 \pi j / K)$ with $\cos (\pi j / k)$ in (3.26) and then set $l=1 / k$. In this way, we formulate our NLEP for the linear stability of a $k$-spike steady-state solution as follows:

Principal Result 3.2 Consider a k-spike steady-state solution to (1.1) on an interval of length 2 subject to Neumann boundary conditions. Then, the NLEP characterizing $\mathcal{O}(1)$ time-scale instabilities of the pattern is

$$
L_{0} \Phi-\chi_{j} w\left(\frac{\int_{-\infty}^{\infty} w^{2} \Phi d z}{\int_{-\infty}^{\infty} w^{3} d z}\right)=\frac{\lambda}{U_{0}-1}, \quad-\infty<z<\infty ; \quad \Phi \rightarrow 0, \quad \text { as } \quad|z| \rightarrow \infty
$$

where $L_{0}$ is defined in (3.5b), and the multipliers $\chi_{j}=\chi_{q j}(\tau \lambda)$ of the non-local term are defined by

$$
\begin{aligned}
& \chi_{j} \equiv 6 \sqrt{2} \beta \pi \omega_{0}^{2} G_{q}(0), \quad G_{q}(0)=\frac{1}{2 \theta_{\lambda}}\left[\tanh \left(\theta_{\lambda} / k\right)+\frac{(1-\cos (\pi j / k))}{\sinh \left(2 \theta_{\lambda} / k\right)}\right]^{-1} \\
& \quad \text { for } j=0, \ldots, k-1
\end{aligned}
$$

Here, $\theta_{\lambda}$ is defined in (3.8), and $w(z)=\sqrt{2} \operatorname{sech} z$ is the homoclinic solution satisfying (2.2).

Moreover, any unstable discrete eigenvalue in $\operatorname{Re}(\lambda)>0$ of the NLEP must be a root of one of the $k$-transcendental equations $\mathcal{F}_{j}(\lambda)=0$, defined by

$$
\mathcal{F}_{j}(\lambda) \equiv \chi_{j}(\tau \lambda)+\frac{\lambda}{U_{0}-1}-3, \quad j=0, \ldots, k-1
$$


We remark that the result (3.28) for the NLEP (3.27) is analogous to that in Lemma 3.1. By re-arranging (3.28), we obtain that $\lambda$ must be a root of

$$
\mathcal{C}_{j}(\tau \lambda)=\mathcal{R}(\lambda), \quad \mathcal{R}(\lambda) \equiv \frac{3\left(U_{0}-1\right)}{3\left(U_{0}-1\right)-\lambda}, \quad j=0, \ldots, k-1,
$$

where $\mathcal{C}_{j}(\tau \lambda)$ is defined by

$$
\mathcal{C}_{j}(\tau \lambda) \equiv \frac{3}{\chi_{j}(\tau \lambda)}=\frac{D \theta_{\lambda}}{\sqrt{2} \pi \beta}\left[\tanh \left(\theta_{\lambda} / k\right)+\frac{(1-\cos (\pi j / k))}{\sinh \left(2 \theta_{\lambda} / k\right)}\right], \quad j=0, \ldots, k-1 .
$$

As derived in Proposition 5.1 of [35] for the related GM model, it is readily shown for $\lambda$ real that $\mathcal{C}_{j}(\tau \lambda)$ is a monotone increasing and concave function of $\lambda$ on $\lambda>0$, with $\mathcal{C}_{j}(0)>0$ and $\left|\mathcal{C}_{j}^{\prime}(\tau \lambda)\right|=\mathcal{O}\left(\tau^{1 / 2}\right) \rightarrow+\infty$ for any $\lambda>0$ as $\tau \rightarrow \infty$. Moreover, the function $\mathcal{R}(\lambda)$ defined in $(3.29 a)$ satisfies $\mathcal{R}(0)=1, \mathcal{R}(\lambda)>0, \mathcal{R}^{\prime}(\lambda)>0$, and $\mathcal{R}^{\prime \prime}(\lambda)>0$, on $0<\lambda<3\left(U_{0}-1\right)$, since $U_{0}>1$. In addition, $\mathcal{R}(\lambda)<0$ on $\lambda>3\left(U_{0}-1\right)$. As such, we conclude that there is a unique root $\lambda^{\star}$ to $(3.29 a)$, satisfying $0<\lambda^{\star}<3\left(U_{0}-1\right)$, whenever $\mathcal{C}_{j}(0)>1$ for some given $j$.

With this criterion, and since $\mathcal{C}_{j}(0)$ in $(3.29 b)$ takes its maximal value over $j=0, \ldots, k-1$ when $j=k-1$, we set $\mathcal{C}_{k-1}(0)>1$, so that with (3.29), the NLEP (3.27) has at least one unstable eigenvalue in $\operatorname{Re}(\lambda)>0$. The assumption $\mathcal{C}_{k-1}(0)>1$ can be rewritten to a condition on $\beta$ leading to an unstable eigenvalue whenever $\beta<\beta_{c k}$, where

$$
\beta_{c k} \equiv \beta_{k}+\frac{1}{\sqrt{2} \pi \omega_{0}} \frac{(1+\cos (\pi / k))}{\sinh \left(2 \omega_{0} / k\right)}
$$

Here, $\omega_{0}=1 / \sqrt{D}$ and $\beta_{k}$ is the existence threshold for the $k$-spike steady-state solution defined in (2.7). Following the terminology in [35], we refer to $\beta_{c k}$ as the competition instability threshold for the spike amplitudes, which arises from the zero-eigenvalue crossing.

Remark that on the range $\beta>\beta_{k}$, we can proceed as in the proof of Proposition 5.3 of [35] to show that there exists a positive minimal value of $\tau$, labelled by $\tau_{H k}$, at which the $\operatorname{NLEP}\left(3.29 a\right.$ ) has a purely complex conjugate pair of eigenvalues $\lambda= \pm i \lambda_{H k}$, with $\lambda_{H k}>0$. This represents the onset of a Hopf bifurcation for the amplitudes of the spikes. In fact, whenever $\mathcal{C}_{j}(0)<1$, the qualitative properties of $\mathcal{C}_{j}(\tau \lambda)$ listed above ensure, for the given $j$, that there are exactly two real eigenvalues on the interval $0<\lambda<3\left(U_{0}-1\right)$ whenever $\tau$ is sufficiently large. On the range $\beta>\beta_{c k}$, for which there are no zero-eigenvalue crossings, the $k$-spike steady-state is linearly stable for $0 \leqslant \tau<\tau_{H k}$ and undergoes a Hopf bifurcation as $\tau$ increases beyond $\tau_{H K}$. This stability information was encoded previously in Figure 1.

\section{Slow transition across stability thresholds: Triggering a finite-time blow-up}

In this section, we examine two scenarios involving slow passage through an instability threshold, which can ultimately trigger a finite-time blow-up behaviour of the spike pattern. Both transitions involve slowly decreasing $\beta$ in time with $\beta=\beta(0)-\sigma t$, where 
$\sigma \ll 1$ and we choose $\beta(0)$ later. We assume that $\mathcal{O}\left(\varepsilon^{2}\right) \ll \sigma \ll \mathcal{O}(1)$ so that the transition is slow with respect to either the $\mathcal{O}(1)$ time-scale of the destabilization of the background state or of the spike amplitudes, as characterized by the NLEP, but is fast with respect to the usual $\mathcal{O}\left(\varepsilon^{2}\right)$ speed characteristic of slowing drifting spikes (cf. [16]).

\subsection{Slow passage through a competition instability}

Our first scenario is to consider a two-spike steady-state solution when $\tau=0$, and to study the effect of a slow transition in $\beta$ below the competition stability threshold $\beta_{c 2}$ (3.30) of the NLEP, and hence, we choose $\beta(0)>\beta_{c 2}$ which triggers a competition instability of the spike amplitudes. To analyse this transition, we proceed as in [1], [9], [21], and [33], by introducing the WKBJ-type perturbation:

$$
v=v_{e}+\phi(x) e^{\sigma^{-1} \Lambda(\sigma t)}, \quad u=u_{e}+\eta(x) e^{\sigma^{-1} \Lambda(\sigma t)},
$$

with $\Lambda(0)=0$ and $\phi \ll 1, \psi \ll 1$. Here, $v_{e}, u_{e}$ is the two-spike steady-state solution. Upon substituting (4.1) into (1.1), and linearizing the resulting system, we obtain that $\phi$ and $\eta$ satisfy

$$
\begin{gathered}
\varepsilon^{2} \phi_{x x}-\left(u_{e}-1\right) \phi+3 v_{e}^{2} \phi-v_{e} \eta=\Lambda^{\prime}(T) \phi, \quad|x| \leqslant 1 ; \quad \phi_{x}( \pm 1)=0, \\
D \eta_{x x}-\left(1+\tau \Lambda^{\prime}(T)\right) \eta=-6 \varepsilon^{-1} \beta(T) v_{e}^{2} \phi, \quad|x| \leqslant 1 ; \quad \eta_{x}( \pm 1)=0,
\end{gathered}
$$

where $\beta(T)=\beta(0)-T$ and $T=\sigma t$ is the new time scale with $\varepsilon^{2} \ll \sigma \ll 1$.

Upon comparing (4.2) with (3.2), we observe that the eigenvalue parameter $\lambda$ in (3.2) is replaced by $\Lambda^{\prime}(T)$. As such, when $\tau=0$, we conclude from the results (3.28) and (3.27b) of the NLEP theory with $k=2, j=1$, and $\tau=0$, that $\Lambda^{\prime}(T)$ satisfies

$$
\frac{\Lambda^{\prime}}{U_{0}-1}=3-\chi_{1}(0), \quad \chi_{1}(0)=3 \sqrt{2} \pi \omega_{0} \beta(T)\left[\tanh \left(\frac{\omega_{0}}{2}\right)+\frac{1}{\sinh \omega_{0}}\right]^{-1}
$$

where $\omega_{0}=1 / \sqrt{D}$. To simplify (4.3), we first use (2.7) with $k=2$, written as

$$
U_{0}-1=\left(\frac{\beta}{\beta_{2}}-1\right)^{-1}, \quad \beta_{2}=\frac{1}{\sqrt{2} \pi \omega_{0}} \tanh \left(\omega_{0} / 2\right)
$$

to reduce (4.3) to

$$
\Lambda^{\prime}=3\left(\frac{\beta}{\beta_{2}}-1\right)^{-1}\left[1-\frac{\beta}{\beta_{2}}\left(\frac{\tanh \left(\omega_{0} / 2\right)}{\tanh \left(\omega_{0} / 2\right)+\operatorname{csch} \omega_{0}}\right)\right] .
$$

Then, we use the identity

$$
\frac{\tanh \left(\omega_{0} / 2\right)}{\tanh \left(\omega_{0} / 2\right)+\operatorname{csch} \omega_{0}}=1-\gamma, \quad \text { where } \quad \gamma \equiv \frac{1}{1+2 \sinh ^{2}\left(\omega_{0} / 2\right)}<1
$$


to express (4.4) in compact form as

$$
\Lambda^{\prime}(T)=-3+\frac{3 \gamma\left[\beta(T) / \beta_{2}\right]}{\left(\beta(T) / \beta_{2}\right)-1}=-3+3 \gamma \frac{\beta(T)}{\beta(T)-\beta_{2}} .
$$

We recall from (2.7) that there is no two-spike steady-state solution when $\beta<\beta_{2}$. Moreover, from (3.30) with $k=2$, the two-spike steady-state is linearly unstable to a competition instability on the range $\beta_{2}<\beta<\beta_{c 2}$ when $\tau=0$. Upon setting $k=2$ in (3.30), we can readily write the competition instability threshold $\beta_{c 2}$ in terms of $\gamma$, as

$$
\beta_{c 2}=\frac{\beta_{2}}{1-\gamma} .
$$

For the slow transition, we let $\beta(T)=\beta(0)-T$, where $T=\sigma t$ with $\sigma \ll 1$, and choose $\beta(0)$ so that $\beta(0)>\beta_{c 2}$. Then, by increasing $T$, we are performing a slow sweep that will eventually cross below the competition instability threshold $\beta_{c 2}$. As characteristic of delayed bifurcation problems ( $\mathrm{cf}$. $[1,9,21,33]$ ), we will show that the instability is triggered not at $\beta_{c 2}$ but at some $\beta^{\star}$ with $\beta^{\star}<\beta_{c 2}$. From (4.1), the criterion for detecting the point at which the instability is triggered is related to determining a time $T^{\star}$ at which $\Lambda\left(T^{\star}\right)=0$. We now show, for arbitrary $\beta(0)>\beta_{c 2}$, that there is a unique $T^{\star}$ with $\beta_{2}<\beta\left(T^{\star}\right)<\beta_{c 2}$ at which the delayed competition instability is triggered. For this, we will need to show that the instability is triggered before $\beta$ passes below the existence threshold $\beta_{2}$.

Without loss of generality, we will set $\Lambda(0)=0$. Our result for $\Lambda(T)$ for $T>0$ is as follows.

Lemma 4.1 Let $\beta(T)=\beta(0)-T$ where $\beta(0)$ is any value satisfying $\beta(0)>\beta_{c 2}$. Then, we have

(i) $\Lambda^{\prime}(0)<0$,

(ii) $\Lambda^{\prime}=0$, when $\beta=\beta_{c 2}$,

(iii) $\exists$ a unique $\beta=\beta^{\star}$, with $\beta_{2}<\beta^{\star}<\beta_{c 2}$, at which $\Lambda=0$.

Here, $\beta^{\star} \equiv \beta\left(T^{\star}\right)=\beta(0)-T^{\star}$, relates $\beta^{\star}$ to the time $T^{\star}$ at which the competition instability is finally triggered.

Proof To prove (i), we use $\beta(0)>\beta_{c 2}=\beta_{2} /(1-\gamma)$, to estimate $1-\frac{\beta_{2}}{\beta(0)}>\gamma$ since $\gamma<1$. Upon using this inequality in (4.6), we find that

$$
\Lambda^{\prime}(0)=-3+3 \gamma \frac{\beta(0)}{\beta(0)-\beta_{2}}<-3+3=0 .
$$

To establish (ii), we set $\Lambda^{\prime}=0$ to obtain the unique root $\gamma \beta=\beta-\beta_{2}$, which yields $\beta=\beta_{c 2} \equiv \beta_{2} /(1-\gamma)$.

This establishes that $\Lambda(0)=0, \Lambda^{\prime}(T)<0$ for $0 \leqslant T<T_{c}, \Lambda^{\prime}\left(T_{c}\right)=0$ where $\beta\left(T_{c}\right)=\beta_{c 2}$, and $\Lambda^{\prime}(T)>0$ for $T>T_{c}$. To prove (iii), it suffices to show that $\Lambda(T) \rightarrow+\infty$ as $\beta \rightarrow \beta_{2}^{+}$. 

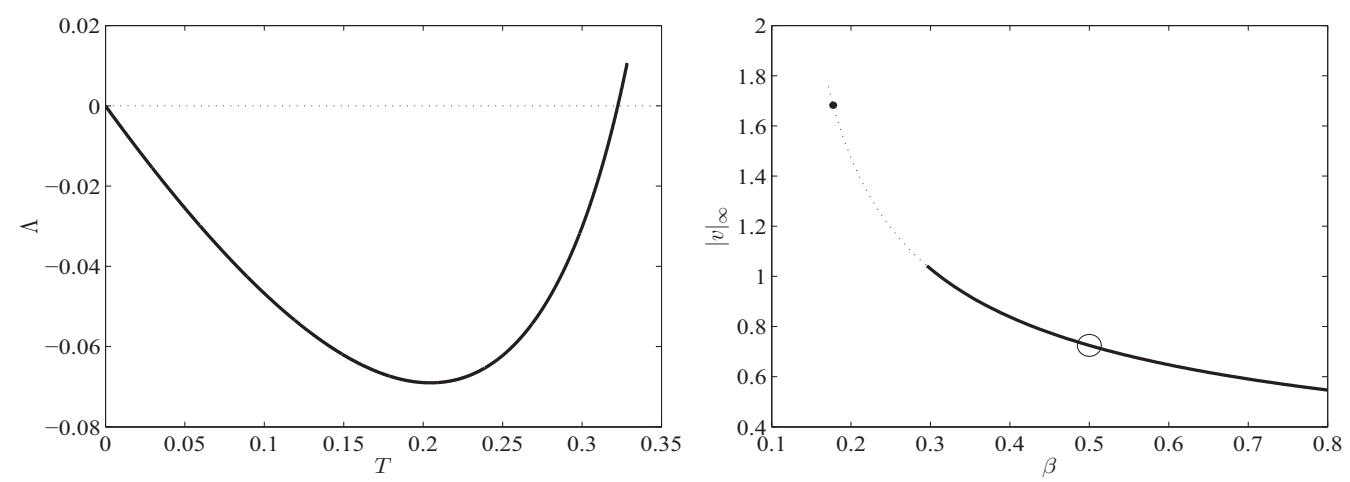

FiguRE 5. Left panel: $\Lambda(T)$ versus $T$, computed from (4.9), for a two-spike steady-state solution with $D=1$ and $\tau=0$ as $\beta=0.5-T$ is slowly swept below the competition instability threshold $\beta_{c 2} \approx 0.296$. The instability is triggered when $\Lambda\left(T^{\star}\right)=0$, which yields $T^{\star} \approx 0.323$ and $\beta\left(T^{\star}\right) \approx$ 0.1768 . Right panel: $|v|_{\infty}=\sqrt{2\left(U_{0}-1\right)}$ versus $\beta$ for a two-spike steady-state with $D=1$. The heavy solid portion is linearly stable for $\tau=0$, while the dashed portion is unstable. The transition occurs at the competition threshold $\beta_{c 2} \approx 0.296$. The open circle indicates the starting point for the slow sweep, while the bullet indicates where the delayed competition instability is finally triggered.

To establish such a result, we determine $\Lambda$ by using (4.6) where we substitute $\beta(T)=$ $\beta(0)-T$. This yields

$$
\Lambda^{\prime}(T)=-3+3 \gamma \frac{\beta(T)}{\beta(T)-\beta_{2}}=-3+3 \gamma\left[1+\frac{\beta_{2}}{\beta(0)-\beta_{2}-T}\right] .
$$

Now, we integrate with respect to $T$, which leads to

$$
\Lambda(T)=-3 T(1-\gamma)-3 \beta_{2} \gamma \log \left(\beta(0)-\beta_{2}-T\right)+c
$$

for some integration constant $c$. Next, we use $\Lambda(0)=0$ which gives $c=3 \beta_{2} \gamma \log \left(\beta(0)-\beta_{2}\right)$, and results in

$$
\Lambda(T)=-3 T(1-\gamma)-3 \beta_{2} \gamma \log \left(1-\frac{T}{\beta(0)-\beta_{2}}\right),
$$

where $0<\gamma<1$ is defined in (4.5). Therefore, $\Lambda \rightarrow+\infty$ as $T$ approaches $\beta(0)-\beta_{2}$ from below, corresponding to $\beta(T) \rightarrow \beta_{2}^{+}$.

In the left panel of Figure 5, we use (4.9) to plot $\Lambda(T)$ versus $T$ for $D=1$ and $\beta(0)=0.5$. For this example, we calculate that $\beta_{2} \approx 0.104, \beta_{c 2} \approx 0.296$, and $\beta^{\star} \approx 0.176$, corresponding to $T^{\star} \approx 0.323$. In the right panel of Figure 5 , we plot the bifurcation diagram of the two-spike steady-state showing the initial point for the slow sweep and the point at which we predict that the competition instability is triggered. From the criterion (3.3) for the stability of the background state, we conclude from Figure 2 that, for this parameter set, the background state is linearly stable throughout the slow sweep. We provide full numerical simulations for the scenario we analysed here in Section 4.3. There, we observe in Experiment 4 that the competition instability first leads to the annihilation of one of the two spikes and can lead to a subsequent finite-time blow-up of the remaining spike. 


\subsection{Slow passage through a Hopf bifurcation}

Next, we consider a one-spike steady-state solution in the shadow limit $D \gg 1$ for a fixed $\tau>0$. We analyse the effect of decreasing $\beta$ slowly in time towards the existence threshold $\beta_{1}$, so that $\beta$ that must cross below the Hopf bifurcation threshold $\beta_{H}=\beta_{1}(1+3 \tau)$, as obtained from (3.15) with $\beta_{1}=1 /(\sqrt{2} \pi)$. In the numerical results shown in Experiment 3 of Section 4.3, this slow sweep initially triggers a time-periodic oscillation of the spike amplitude, which is followed by a finite-time blow-up, see Figure 12.

To analyse this slow passage problem, we proceed as in Section 4.1 and introduce the perturbation (4.1) to obtain the linearized problem (4.2). Now, the NLEP becomes (3.11) where $\lambda$ is to be replaced by $\Lambda^{\prime}(T)$ and for $D \gg 1$, the associated spectral problem is given by (3.14) with $\lambda=\Lambda^{\prime}(T)$. We conclude that $\Lambda^{\prime}(T)$ must satisfy

$$
\left(1+\tau \Lambda^{\prime}\right)=\frac{3 U_{0}}{3\left(U_{0}-1\right)-\Lambda^{\prime}},
$$

which is a quadratic equation. From (2.7), we have $U_{0}-1=\left(\beta / \beta_{1}-1\right)^{-1}$ where $\beta_{1}=$ $1 /(\sqrt{2} \pi)$. Upon using this expression for $U_{0}$, we solve the equation above for $\Lambda^{\prime}$ to obtain

$$
\Lambda^{\prime}=\frac{1}{2 \tau}\left[\left(\frac{3 \tau \beta_{1}}{\beta-\beta_{1}}-1\right) \pm \sqrt{\left(1-\frac{3 \tau \beta_{1}}{\beta-\beta_{1}}\right)^{2}-12 \tau}\right],
$$

where $\beta(T)=\beta(0)-T$. We assume that $\beta(0)>\beta_{H}=\beta_{1}(1+3 \tau)$, and choose $\beta(0)$ such that the term inside the square root in (4.10) is negative. This latter condition holds when

$$
\beta(0)>\beta_{H}, \quad \text { for } \tau \geqslant 1 / 12 ; \quad \beta_{H}<\beta(0)<\beta_{1}\left[1+\frac{3 \tau}{(1-2 \sqrt{3} \tau)}\right], \quad \text { for } 0<\tau<1 / 12 .
$$

Assuming that the condition (4.11) on $\beta(0)$ holds, we separate $\Lambda(T)$ into real and imaginary parts, as $\Lambda=\Lambda_{R}+i \Lambda_{I}$, and obtain from (4.10) that

$$
\Lambda_{R}^{\prime}(T)=\frac{1}{2 \tau}\left(\frac{3 \tau \beta_{1}}{\beta-\beta_{1}}-1\right) .
$$

The criterion for the triggering of an instability is that there exists a unique $T^{\star}$ such that $\Lambda_{R}\left(T^{\star}\right)=0$, with $\Lambda_{R}(T)<0$ on $0<T<T^{\star}$. The following result guarantees the existence of such a $T^{\star}$.

Lemma 4.2 Let $\beta(T)=\beta(0)-T$ where $\beta(0)$ satisfies condition (4.11). Then, for $\operatorname{Re}(\Lambda(T)) \equiv \Lambda_{R}(T)$, we have

(i) $\Lambda_{R}^{\prime}(0)<0$,

(ii) $\Lambda_{R}^{\prime}=0$, when $\beta=\beta_{H}=\beta_{1}(1+3 \tau)$,

(iii) $\exists$ a unique $\beta=\beta^{\star}$, with $\frac{1}{\sqrt{2} \pi} \equiv \beta_{1}<\beta^{\star}<\beta_{H}$, at which $\Lambda_{R}=0$. 

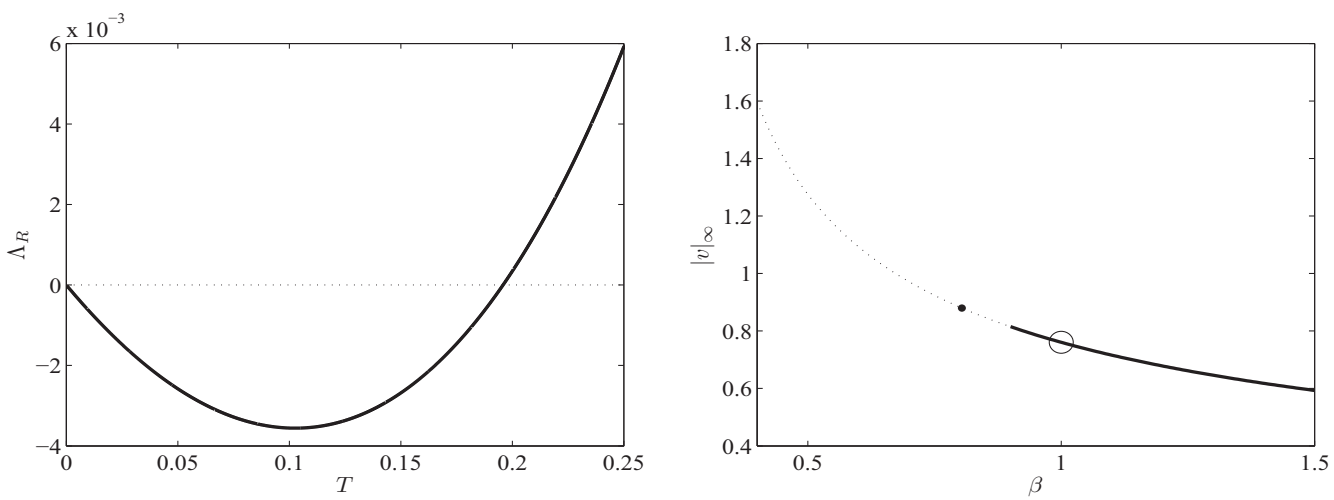

FIGURE 6. Left panel: $\Lambda_{R}(T)$ versus $T$, computed from (4.14), for a one-spike steady-state solution with $D \gg 1$ and $\tau=1.0$ as $\beta=1-T$ is slowly swept below the Hopf instability threshold $\beta_{H} \approx 0.90$. The instability is triggered when $\Lambda_{R}\left(T^{\star}\right)=0$, which yields $T^{\star} \approx 0.191$ and $\beta\left(T^{\star}\right) \approx 0.809$. Right panel: $|v|_{\infty}=\sqrt{2\left(U_{0}-1\right)}$ versus $\beta$ for a one-spike steady-state with $D \gg 1$ and $\tau=1$. The heavy solid portion is linearly stable for $\tau=1$, while the dashed portion for $\beta<\beta_{H}=\beta_{1}(1+3 \tau)$ with $\beta_{1} \approx 0.225$ is unstable due to a Hopf bifurcation. The open circle indicates the starting point for the slow sweep, while the bullet indicates where we predict that the delayed oscillatory instability is finally triggered.

Here, $\beta^{\star} \equiv \beta\left(T^{\star}\right)=\beta(0)-T^{\star}$, relates $\beta^{\star}$ to the time $T^{\star}$ at which the oscillatory instability due to the Hopf bifurcation is finally triggered.

The proof of this result is very similar to that in Lemma 4.1 and is omitted. Instead of (4.9), and with $\Lambda_{R}(0)=0$ when $\beta=\beta(0)$, we readily derive from (4.12) that

$$
\Lambda_{R}(T)=-\frac{T}{2 \tau}-\frac{3 \beta_{1}}{2} \log \left(1-\frac{T}{\beta(0)-\beta_{1}}\right), \quad \beta_{1} \equiv \frac{1}{\sqrt{2} \pi} .
$$

For $\tau=1.0$ and $\beta(0)=1$, in the left panel of Figure 6 , we use (4.14) to plot $\Lambda_{R}(T)$ versus $T$. For this example, we calculate that $\beta_{1} \approx 0.225, \beta_{H} \approx 0.90$, and $\beta^{\star} \approx 0.809$, corresponding to $T^{\star} \approx 0.191$. In the right panel of Figure 6 , we plot the bifurcation diagram of the one-spike steady-state solution showing the initial point for the slow sweep and the point at which we predict that the Hopf bifurcation will be triggered. Since this slow passage problem is for the shadow limit $D \gg 1$, we conclude from Figure 2 that the trivial background state for the activator is always linearly stable.

\subsection{Full numerical results under a slow sweep}

In this section, we show results from full numerical computations on (1.1) using FlexPDE6 [12] that correspond to our linear stability predictions and, in particular, our predictions of triggered competition or oscillatory instabilities under a slow sweep in $\beta$. For one particular example, we will consider the effect of an instability of the trivial background state for the activator $v$.

Experiment 1: We first illustrate an apparent finite-time blow-up of a single spike solution in the shadow limit $D \gg 1$ as $\beta$ is slowly swept below the existence threshold $\beta_{1}$, as 

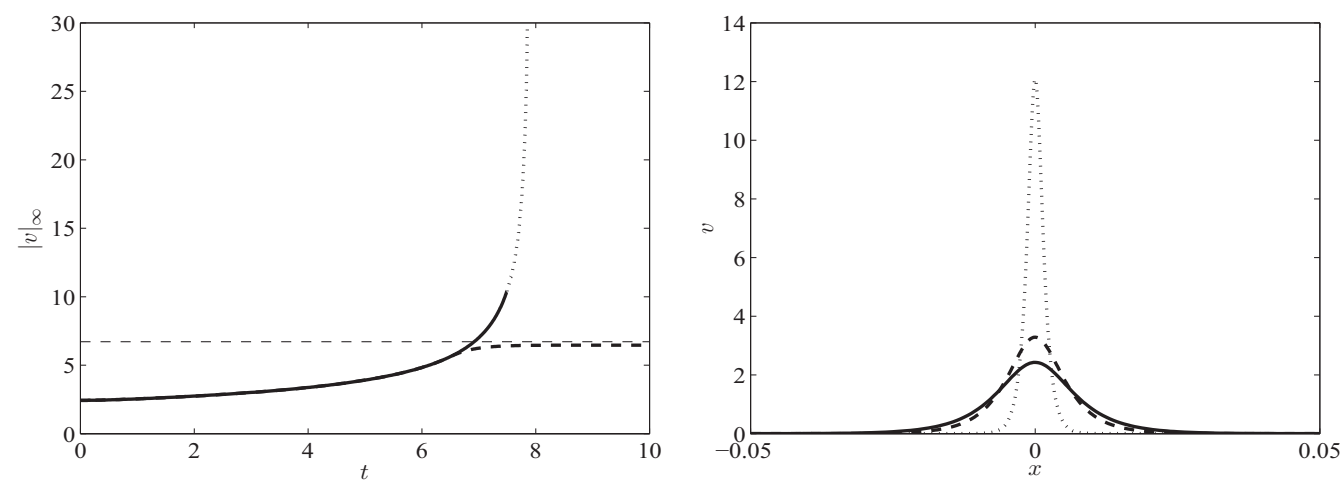

FIgURE 7. Results of Experiment 1. Left panel: The evolution of the spike amplitude as $\beta$ is slowly ramped according to $\beta=\max \left(0.3-\sigma t, \beta_{f}\right)$. When $\beta_{f}>\beta_{1}$ (heavy dashed), the spike amplitude reaches an equilibrium value close to that predicted by the steady state theory of Section 2 (dashed horizontal). When $\beta_{f}<\beta_{1}$, an apparent finite-time blow-up of the amplitude occurs. The heavy solid (heavy dotted) portion indicates the time during which $\beta>\beta_{1}\left(\beta<\beta_{1}\right)$. Right panel: The corresponding spike profiles at times $t=0$ (solid), $t=3.8$ (dashed), and $t=7.6$ (dotted). In both figures, $D=100, \tau=0, \varepsilon=0.01$, and $\sigma=0.01$.

studied in Section 4.2. We take $\tau=0, D=100 \gg 1$, and $\varepsilon=0.01$ in (1.1) and construct a one-spike equilibrium solution as in Section 2 . With $D \gg 1$ and $k=1$, the result (2.8) states that the one-spike steady-state exists for $\beta>\beta_{1}=1 /(\sqrt{2} \pi) \approx 0.225$. For $\beta$, we consider the slow $\operatorname{ramp} \beta=\max \left(0.3-\sigma t, \beta_{f}\right)$, where $\sigma=0.01$. For $\beta_{f}$, we consider two scenarios namely that it is larger than $\beta_{1}$ with $\beta_{f}=\beta_{1}+0.01$ and smaller than $\beta_{1}$ with $\beta_{f}=\beta_{1}-0.01$. For both choices, the evolution of the spike amplitude $|v|_{\infty}$ is given as a function of $t$ in Figure 7 (left) by the thick, dashed $\left(\beta_{f}>\beta_{1}\right)$, or solid $\left(\beta_{f}<\beta_{1}\right)$, curves. In the first scenario where $\beta_{f}>\beta_{1}$, the system reaches an equilibrium with a spike amplitude close to that predicted by the steady state theory of Section 2 (dashed horizontal). In the second scenario, where $\beta_{f}<\beta_{1}$, Figure 7 (left) shows a rapid increase in the spike amplitude beginning when $\beta$ is ramped below $\beta_{1}$. The heavy solid (heavy dotted) portion indicates the time during which $\beta>\beta_{1}\left(\beta<\beta_{1}\right)$. In Figure 7 (right), we show the spike profile at various times over the course of the slow ramp of $\beta$ for the second scenario. Notice that as the spike amplitude grows, the width narrows due to the spatial scaling in (2.1).

To support our conjecture of a finite-time blow-up in the second scenario, we plot in Figure 8 the numerically computed norms $|u|_{\infty}$ and $|v|_{\infty}$ near the apparent singularity time $T \approx 8.1197$. The $\log -\log$ plot in the right panel of Figure 8 supports the scaling law $|u|_{\infty} \sim \mathcal{O}\left((T-t)^{-1}\right)$ and $|v|_{\infty} \sim \mathcal{O}\left((T-t)^{-1 / 2}\right)$ as $t \rightarrow T^{-}$we assume in Section 5.

Experiment 2: Next, we investigate the effects of an instability of the trivial background state for the activator $v$ on the dynamics of a one-spike pattern subject to a slow sweep of $\beta$. Here, we take $D=0.1, \tau=0$, and $\varepsilon=0.02$. For this example, we also need to calculate the competition stability thresholds $\beta_{c 2}$ and $\beta_{c 3}$ for a two- and a three-spike steady-state, and the threshold $\beta_{b}$, obtained from (3.3), at which the background state goes unstable. These thresholds are plotted for a range of $D$ in Figure 9. In particular, for $D=0.1$, we find that $\beta_{c 3} \approx 0.082, \beta_{b} \approx 0.077$, and $\beta_{c 2}=0.071$. Moreover, the existence thresholds 

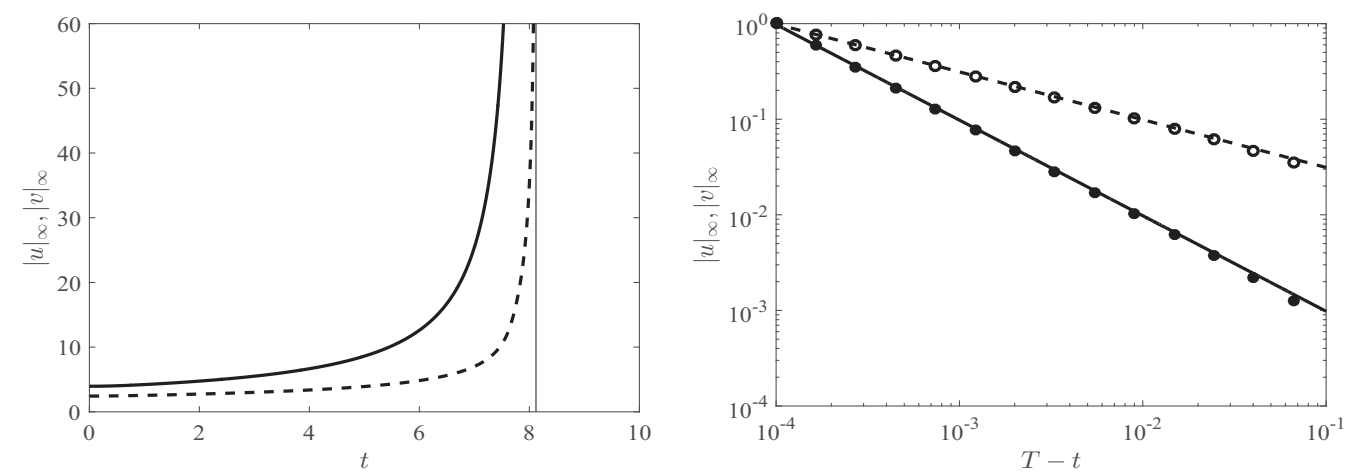

FIGURE 8. Results of Experiment 1. Left panel: Numerical results computed from the PDE model (1.1) supporting the apparent finite-time blow-up conjecture for $|u|_{\infty}$ (heavy solid) and $|v|_{\infty}$ (heavy dashed) as observed in Figure 7 when $\beta=\max \left(0.3-\sigma t, \beta_{f}\right)$, with $\beta_{f}=\beta_{1}-0.01$, is slowly ramped below the existence threshold $\beta_{1}$. The parameters are $D=100, \varepsilon=0.01, \sigma=0.01$, and $\tau=0$. The apparent blow-up time (light solid vertical line) is $T \approx 8.1197$. Right panel: The corresponding $\log -\log$ plot where the solid (dashed) is a plot of $(T-t)^{-1}\left((T-t)^{-1 / 2}\right)$, while the solid (open) circles are (renormalized) data for $|u|_{\infty}\left(|v|_{\infty}\right)$ from the left panel.

given in (2.7) for a $k$-spike steady-state with $k=1,2,3$ are $\beta_{1} \approx 0.079, \beta_{2} \approx 0.0654$, and $\beta_{3} \approx 0.0588$ for $D=0.1$.

We will show that the instability of the background state can initially lead to the formation of additional spikes that have the effect of "absorbing" extra energy when the condition $\beta<\beta_{1}$ would otherwise lead to a finite-time blow-up of a one-spike pattern. However, since $\beta_{1}$ is below the competition thresholds for multi-spike patterns, this absorption is only temporary. The competition instabilities lead to elimination of all but one spike, which is then assured to blow-up when $\beta$ is below the instability threshold of the background state. We illustrate this phenomenon below.

Starting from a one-spike steady-state solution, as constructed in Section 2 and shown in the left panel of Figure 10, we perform a slow sweep of $\beta$ according to $\beta(t)=$ $\max (0.15-\sigma t, 0.065)$ for two values of $\sigma$. Since $\beta(0)>\beta_{b}$, we observe two qualitatively different evolutions depending on $\sigma$. For $\sigma$ only moderately small, the instability of the background state does not have enough time to develop before $\beta$ is ramped to below $\beta_{b}$. The only pattern that exists during the sweep in $\beta$ is a single spike centred at $x=0$. In this scenario, because $\min (\beta(t))<\beta_{1}$, we observe a finite-time blow-up of the one-spike pattern in the same manner as in Experiment 1 (not shown).

However, for $\sigma$ sufficiently small - we take $\sigma=0.001$ - the instability of the background state does have time to grow into a three-spike pattern as shown in the centre and right panels of Figure 10 before $\beta$ crosses below the background instability threshold $\beta_{b}$. However, once $\beta$ is ramped below $\beta_{c 3}$, the competition instability eliminates the middle spike, leaving a two-spike quasi-equilibrium pattern as shown in the left panel of Figure 11. A further decrease in $\beta$ triggers another competition instability, leaving a one-spike quasiequilibrium pattern as shown in the centre panel of Figure 11. The process by which this off-centred quasi-equilibrium spike then apparently undergoes a finite-time blow-up is the same as shown below in Experiment 4. 


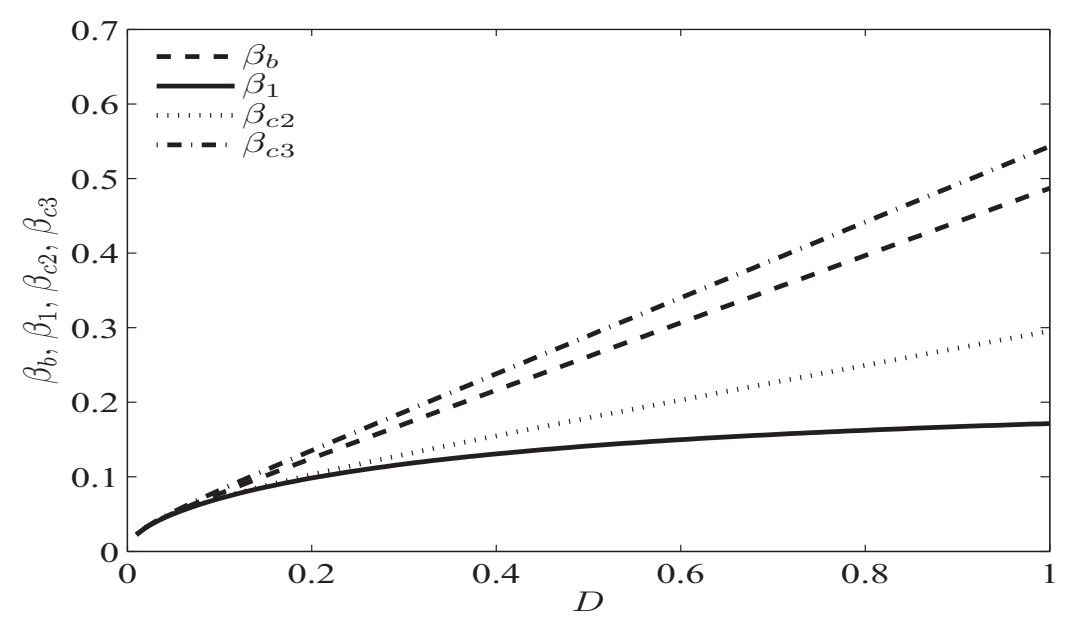

FIGURE 9. Plot of the competition instability thresholds $\beta_{c 2}$ and $\beta_{c 3}$ for two-spike and three-spike steady-state solutions versus $D$, together with the instability threshold $\beta_{b}$ of the trivial background state for a one-spike solution. The one-spike existence threshold $\beta_{1}$ is always below the two competition thresholds $\beta_{c k}$ for $k=2,3$. Therefore, if $\beta$ is slowly ramped to below $\beta_{1}$, a finite-time blow-up of a one-spike pattern is assured, no matter how slowly the sweep is performed.
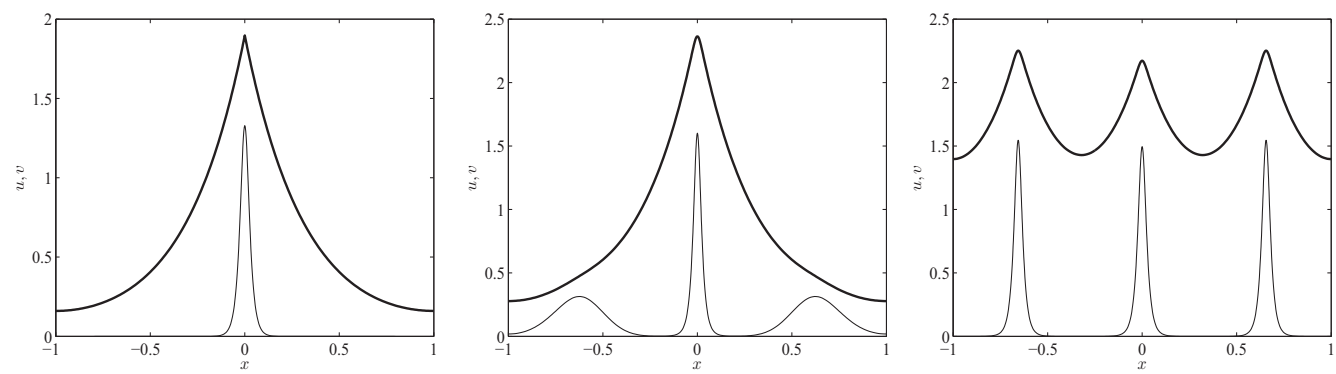

FIGURE 10. Results of Experiment 2 where $v$ is plotted (thinner solid) and $u$ (thick solid). Left panel $(t=0, \beta=0.15)$ : A one-spike equilibrium initial condition. Centre panel $(t=20, \beta=0.13)$ : The instability of the background state results in the growth of two bumps on either side of the spike. Right panel $(t=40, \beta=0.11)$ : The two small bumps develop into spikes located at equilibrium locations $x= \pm 2 / 3$. In all figures, $\beta$ is above the instability threshold $\beta_{b}=0.077$ of the background state. Here, $D=0.1, \tau=0, \varepsilon=0.02$, and $\sigma=0.001$. Solid: $v$, heavy solid: $u$.

This experiment should be compared to Experiment 5.2(a) of [19], where $\beta$ was also ramped past the one-spike existence threshold. In the process of the sweep, dynamics similar to Figure 10 (centre) developed, where two bumps were formed on either side of the original spike. However, instead of evolving close to a three-spike equilibrium, the pattern evolved to a constant steady state. This is in contrast to the process described above, where a series of unstable intermediate states gives way to a one-spike pattern that has an apparent finite-time blow-up.

Experiment 3: In this experiment, we consider a one-spike pattern with $\tau=1, \varepsilon=0.01$, and with $D \gg 1$ in (1.1). We aim to illustrate the theory of Section 4.2, and in particular the example of Figure 6, for slow passage through a Hopf bifurcation threshold. We 

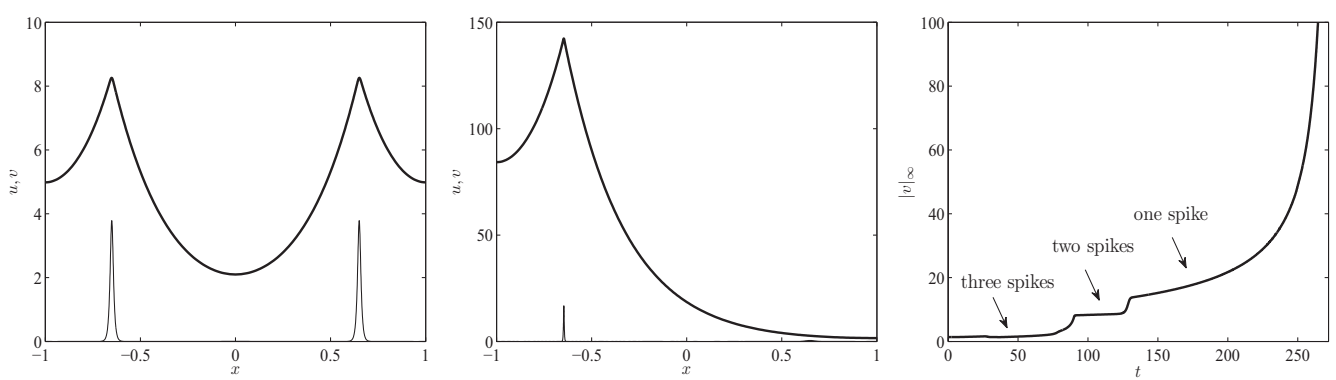

FIGURE 11. Continuation of the evolution begun in Figure 10 (left) of the results of Experiment 2 where again $v$ is plotted thinner and $u$ thicker. Left panel $(t=77.5, \beta=0.0725)$ : Two-spike quasi-equilibrium pattern after the middle spike in Figure 10 (right) has been eliminated due to a competition instability. Centre panel $(t=105, \beta=0.045)$ : A one-spike quasi-equilibrium solution with an off-centred spike after the right spike in the left panel has been eliminated. Right panel: Evolution of $|v|_{\infty}$ in time. Spike elimination events cause the heights of the remaining spikes to increase. Rapid increase of the amplitude of the final remaining spike suggests a finite time blow-up. The background state is stable in the left and centre figures. Here, $D=0.1, \tau=0, \varepsilon=0.02$, and $\sigma=0.001$.

begin with a slight perturbation of a one-spike steady-state as constructed in Section 2 . With $\tau=1$ and $D \gg 1$, we obtain from (3.15) that the Hopf bifurcation threshold for $\beta$ is $\beta_{H} \approx 0.9$. To illustrate the theory presented in Figure 6 , we perform the slow ramp $\beta(T)=1-T$, where $T=0.002 t$. The resulting deviation of the spike amplitude from its steady-state value is shown by the heavy solid curve against $\beta(T)$ in the left panel of Figure 12. For comparison against the theory, we also plot in this figure in light solid the perturbation (4.1) from the stationary solution which is the quantity $\pm C \exp (\Lambda / 0.002)$ for some chosen $C$, where $\Lambda$ is determined from (4.14).

The one-spike steady-state solution is stable during the time that $\beta>\beta_{H}$. This is consistent with Figure 12, where we initially observe decaying oscillations in the spike amplitude. However, once $\beta$ is slowly decreased below $\beta_{H} \approx 0.9$ (dashed vertical), the Hopf bifurcation threshold for the unramped problem is crossed and the amplitude oscillations begin to increase. The delay in the onset of the Hopf bifurcation results from the time needed in order for the oscillations to recover their strength lost during the stable phase of the ramp. We observe that the value of $\beta$ at which the amplitude of oscillations returns to its original value (dotted horizontal) is $\beta\left(T^{*}\right) \approx 0.788$. This is in excellent agreement with the NLEP theory of Section 4.2 , which predicts a value of $\beta\left(T^{*}\right) \approx 0.809$. In the right panel of Figure 12, we show that the delayed Hopf bifurcation results in fully non-linear oscillations, leading to an apparent finite-time blow-up in the spike amplitude before $\beta$ reaches the one-spike existence threshold $\beta_{1} \approx 0.225$. We note that the right panel of Figure 12 is a plot of the actual spike amplitude $|v|_{\infty}$; no steady-state value has been subtracted from it.

Experiment 4: For our final experiment, we consider a two-spike solution with $\tau=0$, $\overline{D=1 \text {, and } \varepsilon}=0.002$ in (1.1). We aim to illustrate the theory of Section 4.1 and in particular the example of Figure 5 for slow passage through a competition instability threshold. We begin with an odd perturbation of a two-spike equilibrium solution with 

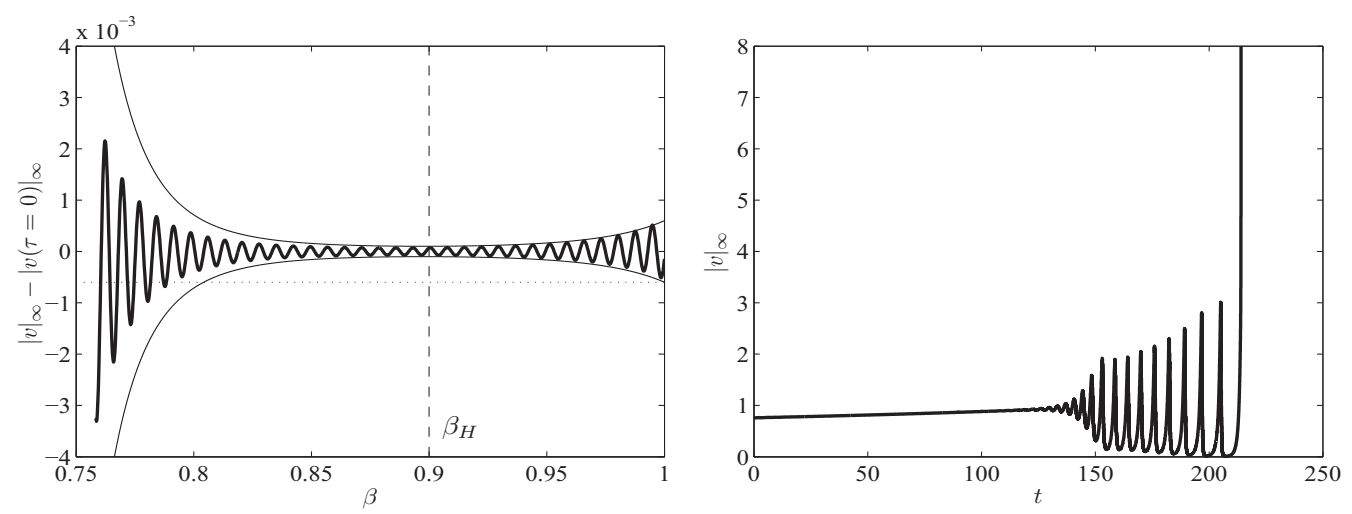

FIgURE 12. Results of Experiment 3. Left panel: The deviation of the spike amplitude from its steady-state value (heavy solid curve) as $\beta$ is slowly ramped according to $\beta(\sigma t)=1-\sigma t$. The thinner solid curve is the quantity $\pm C \exp (\Lambda / \sigma)$ for some chosen $C$, with $\Lambda$ given by (4.14). When $\beta$ is above the Hopf bifurcation threshold $\beta_{H}$ (dashed vertical line), the one-spike steady-state is stable, causing oscillations in the deviation to decay. When $\beta<\beta_{H}$, the Hopf mode is unstable so that the oscillations grow. The bifurcation is fully triggered when the oscillations return to their original magnitude (dotted horizontal line). This occurs at $\beta\left(T^{*}\right) \approx 0.788$, in excellent agreement with the theoretical value of $\beta\left(T^{*}\right) \approx 0.809$. Right panel: The ensuing fully non-linear oscillations of the spike amplitude $|v|_{\infty}$ leading to an apparent finite-time blow-up before $\beta$ reaches the existence threshold $\beta_{1} \approx 0.225$. In both figures, $D=100, \tau=1, \varepsilon=0.01$, and $\sigma=0.002$.

spikes centred at $x= \pm 0.5$. This perturbed solution is shown in the left panel of Figure 13 . For $D=1$, we obtain from the result of the NLEP analysis (3.30) that the competition instability threshold is $\beta_{c 2} \approx 0.296$. To best illustrate the theory, we choose the perturbation to be the eigenfunction corresponding to a competition instability for $\beta=0.28<\beta_{c 2}$. We begin with $\beta=\beta(0)=0.5>\beta_{c 2}$ so that the two-spike pattern is initially stable. We then slowly $\operatorname{ramp} \beta$ according to $\beta=0.5-T$, where $T=0.02 t$. For the time during which $\beta>\beta_{c 2}$, the pattern is stable, leading to a decay of the odd perturbation. This is observed in the centre panel of Figure 13, where we plot the difference in spike amplitudes (circles) normalized to have an initial value of unity as a function of $\beta(T)$. For comparison, the solid curve in the centre panel of Figure 13 is the quantity $\exp (\Lambda(\beta) / 0.02)$, here $\Lambda(\beta)$ evolves as in equation (4.9). We observe excellent agreement in the rate of the initial decay.

When $\beta$ is ramped below $\beta_{c 2} \approx 0.296$, the amplitude difference begins to increase as the competition threshold is slowly passed. We again observe excellent agreement between $\beta$ values for which this occurs numerically and the corresponding NLEP prediction. For all $\beta<\beta_{c 2}$, the two-spike equilibrium is unstable to an $\mathcal{O}(1)$ competition instability. This is reflected by the increasing normalized amplitude difference in the centre panel of Figure 13. It results in a one-spike pattern with a spike at some point $x=x_{0}$ as in Figure 13 right panel, after one of the spikes has been eliminated. By the convention of the delayed bifurcation analysis in Section 4.1, we consider the competition instability fully triggered when the normalized amplitude difference reaches its initial value of 1 . This occurs when $\beta \approx 0.187$, as compared to the theoretically predicted value of $\beta \approx 0.176$. We conjecture that this slight discrepancy is due to the accumulation of numerical errors, as 

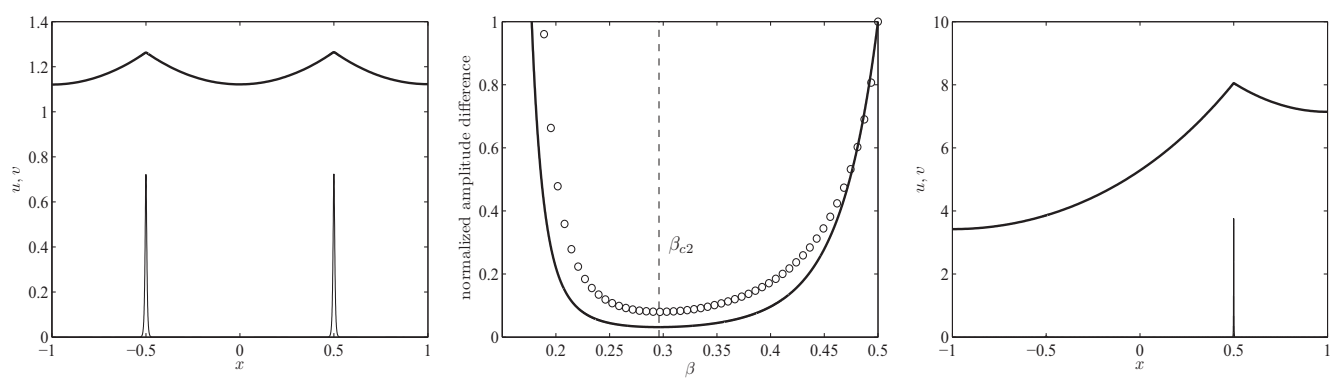

FIgURE 13. Results of Experiment 4. Left panel: A two-spike steady-state solution. Centre panel: The normalized difference in spike amplitudes as $\beta$ is slowly ramped according to $\beta(\sigma t)=0.5-\sigma t$. The light solid is the quantity $\exp (\Lambda / \sigma)$, where $\Lambda$ is obtained from (4.9). When $\beta$ is above the two-spike competition threshold $\beta_{c 2}$ (dashed vertical), the two-spike pattern is stable, resulting in a decay in the amplitude difference. When $\beta$ is ramped below $\beta_{c 2}$, the amplitude difference begins to grow. The competition instability is fully realized when the normalized amplitude difference returns to 1 , occurring at $\beta\left(T_{\text {num }}^{*}\right) \approx 0.188$, which is close to the theoretically predicted value of $\beta\left(T_{\text {asymp }}^{*}\right) \approx 0.177$ given in Figure 5 . Here, $T_{\text {num }}^{*} \approx 15.6$, and $T_{\text {asymp }}^{*} \approx 16.15$. Right panel: The resulting quasi-equilibrium one-spike pattern after the left spike has been eliminated. In all figures, $D=1, \sigma=0.02, \tau=0$, and $\varepsilon=0.002$. Solid: $v$, heavy solid: $u$.

we observe full agreement in both the initial decay (Figure 13 (centre panel)) as well as initial growth rates when $\beta<\beta_{c 2}$ (not shown).

The growth of the competition instability for $\beta<\beta_{c 2}$ does not saturate, but instead continues until only one spike remains in a quasi-equilibrium state, as shown in the right panel of Figure 13. This remaining spike then drifts on an $\mathcal{O}\left(\varepsilon^{2}\right)$ time-scale towards its equilibrium location $x=0$ while its amplitude evolves accordingly. The differentialalgebraic system governing these dynamics has been derived in many past works through application of a Fredholm alternative (e.g., [16]), and will not be repeated here. We instead focus on the possibility of a finite time blow-up of the amplitude of the remaining spike triggered by the slow drift. To illustrate this phenomenon, we follow the procedure of Section 2 to obtain that a single quasi-equilibrium spike centred at $x=x_{0}$ exists only if $\beta>\beta_{q 1}$, where $\beta_{q 1}$ is given by

$$
\beta_{q 1}=\frac{\tanh \left(\omega_{0}\left(1+x_{0}\right)\right)+\tanh \left(\omega_{0}\left(1-x_{0}\right)\right)}{2 \sqrt{2} \pi \omega_{0}}, \quad \omega_{0}=1 / \sqrt{D} .
$$

For $D=1$, a plot of (4.15) versus $x_{0}$ is shown in Figure 14, and this result agrees with the existence threshold $\beta_{1}$, defined in (2.7) with $k=1$, when $x_{0}=0$.

Because $\beta_{q 1}$ is a decreasing function of $x_{0}$, there are three different scenarios for the fate of the remaining spike. If the $\operatorname{ramp}$ of $\beta$ is terminated at some $\beta_{f}>\beta_{1}$ (i.e., $\left.\beta=\max \left(\beta_{0}-T, \beta_{f}\right)\right)$, the spike will simply drift to $x=0$ and remain there for all time. If $\beta_{f}<\beta_{q 1}(0.5)$, the amplitude of the remaining spike will blow-up in finite time before any substantial drift in location occurs. If $\beta_{q 1}(0.5)<\beta_{f}<\beta_{1}$, such as shown in Figure 14, the spike drifts towards $x=0$ until the drift triggers a finite time blow-up. We illustrate this phenomenon in Figure 15, where we set $\beta_{f}=0.1589$ so that $x_{0}^{*} \approx 0.42$ where $\beta_{q_{1}}\left(x_{0}^{*}\right)=\beta_{f}$. In the left panel of Figure 15, we plot $T=\sigma t$ as a function of the location $x_{0}$ of the right 


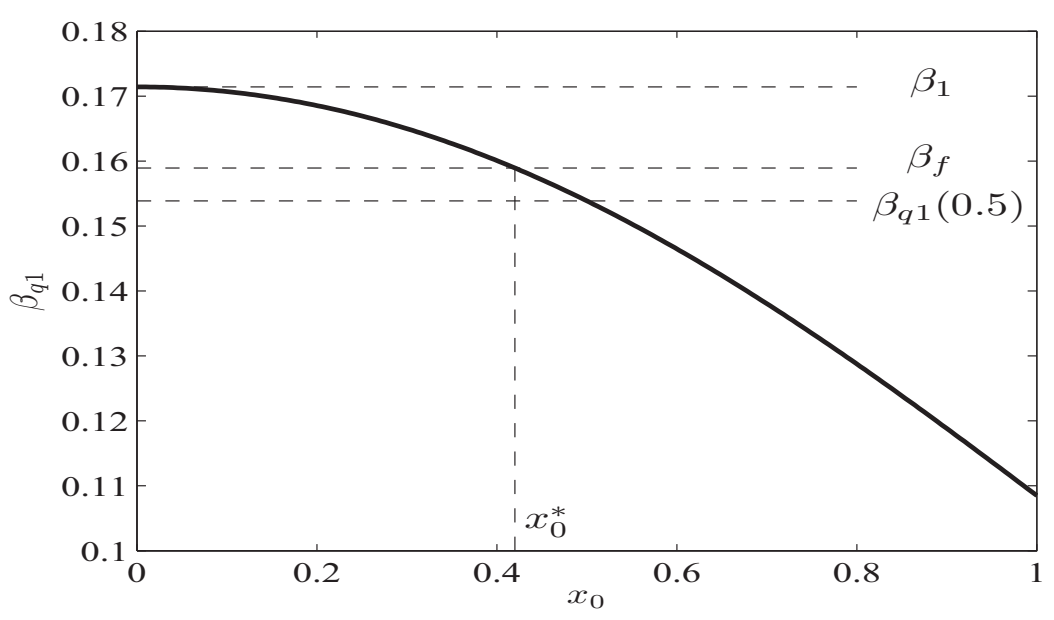

FIGURE 14. Existence threshold $\beta_{q 1}\left(x_{0}\right)$ in (4.15) of a quasi-equilibrium one-spike pattern centred at $x=x_{0}$ for $D=1$. When $\beta_{q 1}(0.5)<\beta_{f}<\beta_{1}$, a finite time blow-up of a single quasi-equilibrium spike is triggered by the slow spike drift towards the origin; it is initiated when the spike location reaches $x_{0}=x_{0}^{*}$.

spike. In the computations, we have taken $\sigma=0.004$. In the right panel of Figure 15, we show the corresponding evolution of the logarithm of the amplitude of the right spike as function of $T=\sigma t$. During the time over which the competition instability eliminates the other spike located at $x=-0.5$, the rightmost spike is essentially stationary. In the right panel of Figure 15, we show the corresponding evolution of the logarithm of the amplitude of the right spike. As the competition mode grows, so does the amplitude of the right spike. Once the left spike is eliminated, the right spike drifts slowly towards $x=0$. The amplitude grows correspondingly during this time. However, once the spike location reaches near $x_{0}=x_{0}^{\star} \approx 0.42$, we observe that the spike ceases its slow drift; quasi-equilibrium theory is no longer valid in this regime. Simultaneously, we see a rapid increase in the amplitude of the spike, indicative of a finite-time blow-up.

\section{The blow-up profile for the non-local Ginzburg-Landau model}

In the numerical simulations of the previous section Section 4.3, finite-time blow-up was observed in several of the Experiments. Here, we focus on the shadow limit $D \gg 1$ as studied in Experiment 1, and hence, we analyse the existence of a solution that blows up in finite time to the non-local GL model (1.3). We assume the finite-time singularity to be positioned at $x=x_{0}$, for some constant $x_{0} \in(-1,1)$, and to occur at blow-up time $t=T<\infty$. We will show that this blow-up solution exists only if $\beta<\beta_{1}$, where $\beta_{1}=1 /(\sqrt{2} \pi)$ is the existence threshold of a one-spike steady-state solution.

To study the behaviour of the blow-up profile near the singularity, we rescale space, time, and $v$ by factors of a suitably chosen norm of the solutions, denoted by $L(t)$, which blows up at the singularity. This rescaling is motivated by the invariance present in the equation $u_{t}=\Delta u+u^{3}$. Here, we use the dynamical rescaling introduced in [11] for this 

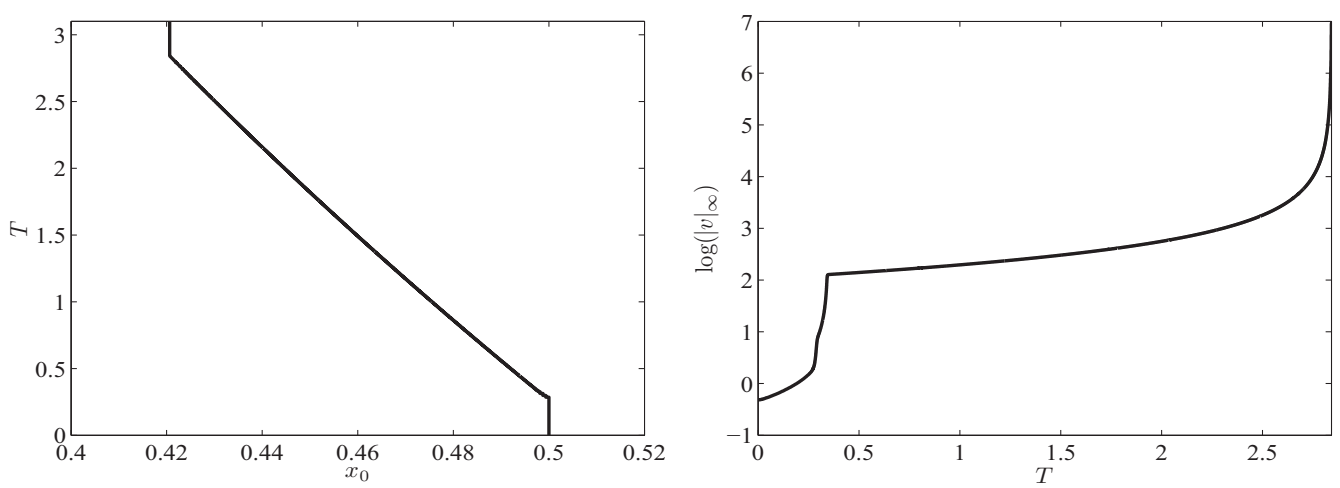

FIgURE 15. Results of Experiment 4. Left panel: Values of $T=\sigma t$ as a function of the location of the right spike. The right spike initially stays at $x_{0}=0.5$ as the competition instability develops. Once the left spike is eliminated, the right spike begins to drift slowly towards $x=0$. During this drift, the quasi-equilibrium spike-existence threshold is reached near $x_{0} \approx 0.42$. Right panel: The corresponding evolution of the logarithm of the spike amplitude. The rapid increase near $T \approx 2.84$ suggests that the finite time blow-up coincides with the spike's approach to $x=0.42$. Here, $D=1$, $\sigma=0.004, \tau=0$, and $\varepsilon=0.002$.

equation, which is defined by

$$
y \equiv \frac{x-x_{0}}{\varepsilon L(t)}, \quad s=-\log (T-t), \quad L(t) \equiv \sqrt{T-t}, \quad v(x, t)=\frac{1}{L(t)} w(y, s) .
$$

Note that $L$ is indeed chosen such that $L(t) \rightarrow 0$ as $t \rightarrow T^{-}$.

This rescaling guarantees that the rescaled problem is no longer singular and equation (1.3) becomes

$$
w_{s}=w_{y y}-\frac{1}{2}\left(w+y w_{y}\right)+e^{-s} w+w^{3}-\beta w \tilde{I}(w), \quad \tilde{I}(w) \equiv \int_{-\varepsilon^{-1}\left(1+x_{0}\right) e^{\frac{s}{2}}}^{\varepsilon^{-1}\left(1-x_{0}\right) e^{\frac{s}{2}}} w^{3} d y .
$$

Since $t \rightarrow T^{-}$corresponds to $s \rightarrow \infty$, we will first determine the limiting behaviour of the solution to (5.2) as $s \rightarrow \infty$. This limiting steady-state solution $W(y)$ satisfies

$$
W_{y y}-\frac{1}{2}(y W)_{y}-\beta W I(W)+W^{3}=0, \quad I(W) \equiv \int_{-\infty}^{\infty} W^{3} d y .
$$

We will look for a positive solution to (5.3) with one maximum at the origin, so that $W_{y}=0$ at $y=0$ and $W \rightarrow 0$ as $y \rightarrow \pm \infty$. Observe that solutions to this equation are symmetric.

Note that for every solution $W$ to equation (5.3) the product of $\beta$ and $I(W)$ is constant, and therefore, the solution branch of (5.3) can be parameterized by this product that we denote by $c$. In terms of the solution, $\beta=\beta(c)$ is then given by

$$
\beta(c) \equiv \frac{c}{\int_{-\infty}^{\infty}[W(y)]^{3} d y},
$$



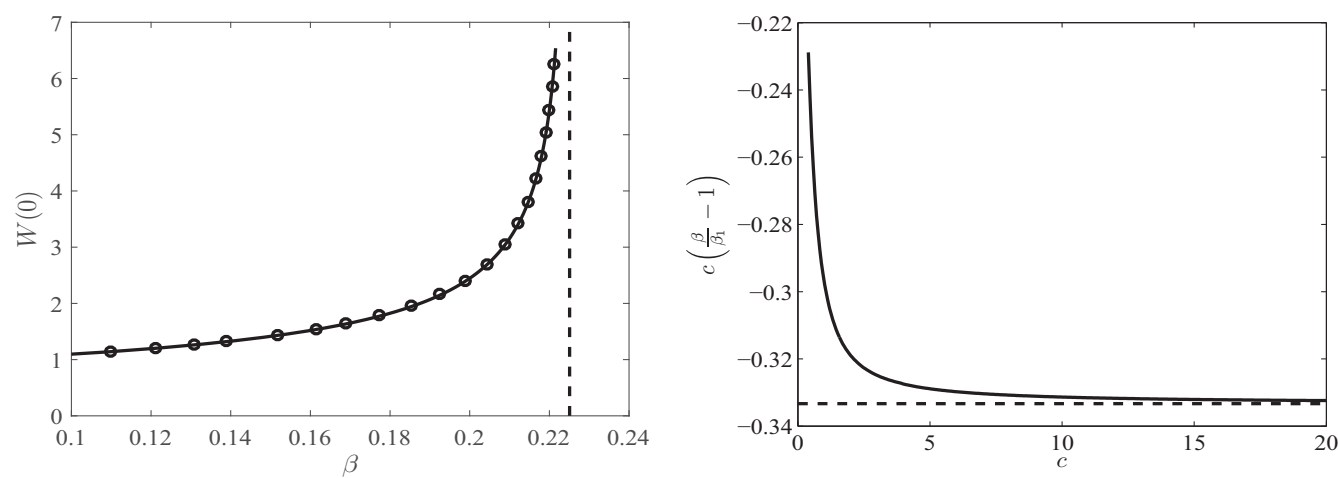

FIGURE 16. Left panel: $W(0)$ versus $\beta$ as computed numerically (solid curve) from (5.4) in terms of the parameter $c$. The discrete points are the asymptotic result $W(0)=\sqrt{2 c}$ versus $\beta=\beta_{1}\left(1-\frac{1}{3 c}\right)$. As $c \rightarrow \infty$, we have $\beta \rightarrow \beta_{1}=1 /(\sqrt{2} \pi)$ from below, where $\beta_{1}$ is the existence threshold for a onespike solution (vertical dashed line). Right panel: validation of the asymptotics $\beta \sim \beta_{1}(1-1 /(3 c)$ ) as $c \rightarrow+\infty$. We plot $c\left(\beta / \beta_{1}-1\right)$ versus $c$, where $\beta$ is computed numerically from (5.4). This this quantity tends to the theoretically predicted value of $-1 / 3$ as $c \rightarrow \infty$ given by the horizontal dashed line.

and the solutions satisfy

$$
W_{y y}-\frac{1}{2}(y W)_{y}-c W+W^{3}=0
$$

where $W$ has a maximum at the origin $\left(W_{y}=0\right.$ at $\left.y=0\right)$, and $W \rightarrow 0$ as $y \rightarrow \pm \infty$. To gain insight about the behaviour of solutions, we first solve equation (5.4b) numerically as $c$ is varied, and use $(5.4 a)$ to obtain $\beta$. This results in the solution branch where the solution at the origin, $W(0)$, is given versus $\beta$ as shown in the left panel of Figure 16.

We now observe that $\beta$ tends from below to the existence threshold $\beta_{1}=1 /(\sqrt{2} \pi)$ of a one-spike solution - the vertical asymptote in the left panel of Figure $16-$ as $c \rightarrow+\infty$ in $(5.4 b)$. As expected, this implies that there does not exist a blow-up solution in the range of $\beta, \beta>\beta_{1}$ where a one-spike steady-state solution exists.

To show that this is indeed the case, we now analyse equation $(5.4 b)$ for $c \gg 1$ to construct such a blow-up solution for $\beta<\beta_{1}$. For this, we study the equation in various regions and assume that the main part of the solution lies around the origin since the solution decays to zero as $y \rightarrow \pm \infty$. In this region around $y=0$, the solution has a maximum (at $y=0$ ), and therefore, we call this the bump region.

First, we zoom into the origin and rescale $y$ and $W$ by introducing the local variables $z$ and $\mathcal{W}$ by

$$
z=\sqrt{c} y, \quad \mathcal{W}(z)=c^{-1 / 2} W(z / \sqrt{c}) .
$$

Upon substituting (5.5) into equation $(5.4 b)$, we obtain that $\mathcal{W}(z)$ satisfies

$$
\mathcal{W}_{z z}-\frac{1}{2 c}\left[\mathcal{W}-z \mathcal{W}_{z}\right]-\mathcal{W}+\mathcal{W}^{3}=0
$$

We define the bump region as the region where $|z| \ll \sqrt{c}$, and hence $|y| \ll 1$, so that the second term in this equation is higher order compared to the rest of the terms. We 
then expand the solution in terms of the small parameter $\frac{1}{c}$

$$
\mathcal{W}=\mathcal{W}_{0}+\frac{1}{c} \mathcal{W}_{1}+\cdots,
$$

and obtain to leading-order that

$$
\mathcal{W}_{0 z z}-\mathcal{W}_{0}+\mathcal{W}_{0}^{3}=0
$$

so that

$$
\mathcal{W}_{0}=\sqrt{2} \operatorname{sech} z
$$

At next order, we obtain that $\mathcal{W}_{1}$ satisfies

$$
L_{0} \mathcal{W}_{1} \equiv \mathcal{W}_{1 z z}-\mathcal{W}_{1}+3 \mathcal{W}_{0}^{2} \mathcal{W}_{1}=\frac{1}{2}\left[\mathcal{W}_{0}+z \mathcal{W}_{0 z}\right]
$$

where $L_{0}$ was introduced in $(3.5 b)$.

Although the two-term approximation (5.7) does not provide a uniformly valid characterization of $W(y)$ for $y \gg 1$, we show below in a WKBJ analysis of the far-field of the blow-up profile that our two-term result (5.7) is sufficient to determine a two-term approximation of the integral in $(5.4 a)$ defining $\beta$. Before we turn to this WKBJ-approach, we first determine this approximation of $\beta$.

Upon using the expansion (5.7) and the rescaling (5.5), we find that

$$
\int_{-\infty}^{\infty} W^{3} d y=c \int_{-\infty}^{\infty}\left(\mathcal{W}_{0}+c^{-1} \mathcal{W}_{1}+\cdots\right)^{3} d z \sim c\left[\int_{-\infty}^{\infty} \mathcal{W}_{0}^{3} d z+3 c^{-1} \int_{-\infty}^{\infty} \mathcal{W}_{0}^{2} \mathcal{W}_{1} d z\right]
$$

The first term in this expansion can be determined by using the sech-solution (5.8) which yields $\int_{-\infty}^{\infty} \mathcal{W}_{0}^{3} d z=\sqrt{2} \pi$.

Next, we determine the second integral in expression (5.10). We use equation (5.9) and the identity $L_{0} \mathcal{W}_{0}^{2}=3 \mathcal{W}_{0}^{2}$ (see Lemma 2.3 of [25]), together with integration by parts and the decay of $\mathcal{W}_{0}$ at infinity, to obtain

$$
\begin{aligned}
\int_{-\infty}^{\infty} \mathcal{W}_{0}^{2} \mathcal{W}_{1} d z & =\frac{1}{3} \int_{-\infty}^{\infty}\left(L_{0} \mathcal{W}_{0}^{2}\right) L_{0}^{-1}\left[\frac{1}{2}\left(\mathcal{W}_{0}+z \mathcal{W}_{0 z}\right)\right] d z \\
& =\frac{1}{6} \int_{-\infty}^{\infty} \mathcal{W}_{0}^{2}\left(\mathcal{W}_{0}+z \mathcal{W}_{0 z}\right) d z \\
& =\frac{1}{6} \int_{-\infty}^{\infty}\left(\mathcal{W}_{0}^{3}+\frac{1}{3} z\left(\mathcal{W}_{0}^{3}\right)_{z}\right) d z=\frac{1}{9} \int_{-\infty}^{\infty} \mathcal{W}_{0}^{3} d z=\frac{\sqrt{2} \pi}{9}
\end{aligned}
$$

Upon substituting both of these results into (5.4a), we obtain that, to leading order, for $c \gg 1$, we have

$$
\beta=\frac{c}{c \sqrt{2} \pi+\frac{\sqrt{2} \pi}{3}}=\frac{1}{\sqrt{2} \pi}\left[1-\frac{1}{3 c}\right]=\beta_{1}\left[1-\frac{1}{3 c}\right], \quad \text { as } \quad c \rightarrow \infty,
$$




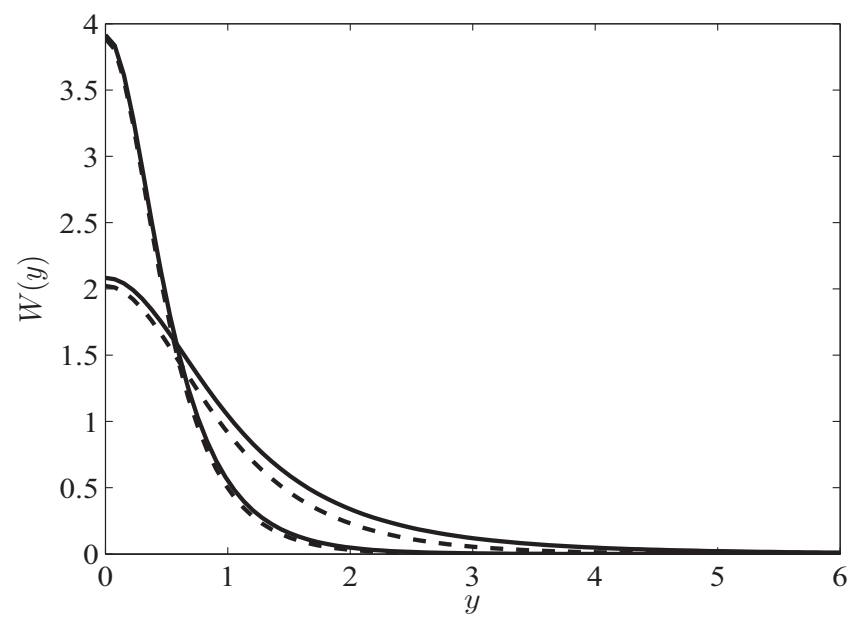

FIGURE 17. The solid curves are the full numerical solution to $(5.4 b)$ for $c=2.0455(\beta \approx 0.1899)$ and for $c=7.558(\beta \approx 0.21508)$. The curve with the larger $W(0)$ value corresponds to $c=7.558$. The dashed curves are the leading-order bump solution (5.12). This bump solution is essentially indistinguishable from the numerical solution for $c=7.558$, and for $c=2.0455$.

where $\beta_{1}=1 /(\sqrt{2} \pi)$ is the existence threshold of a one-spike steady-state solution.

In Figure 16, we compare this asymptotic result with the one from numerical simulations. In the right panel of Figure 16, we plot $c\left(\frac{\beta}{\beta_{1}}-1\right)$ versus $c$, where $\beta$ is computed numerically from (5.4a). This plot corresponds nicely to the asymptotic prediction of (5.11) that $c\left(\frac{\beta}{\beta_{1}}-1\right) \rightarrow-1 / 3$ as $c \rightarrow \infty$. In the left panel of Figure 16, we plot the curve $W(0)$ versus $\beta$ which are both parameterized by $c$ as $W(0)=\sqrt{2 c}$ and $\beta=\beta_{1}\left(1-\frac{1}{3 c}\right)$, by the discrete points. These agree rather well with the full numerical result (solid line) even when $\beta$ is not close to the existence threshold $\beta_{1}$.

We summarize the results so far as follows. We showed for $|y| \ll 1$ that as $c \rightarrow \infty$, or equivalently as $\beta \rightarrow \beta_{1}^{-}$, the leading order solution in the bump region is given by

$$
W(y)=\sqrt{2 c} \operatorname{sech}(\sqrt{c} y), \quad \text { where } c=\left[3\left(1-\frac{\beta}{\beta_{1}}\right)\right]^{-1} .
$$

We plot this leading order bump solution (5.12) in dashed curves in Figure 17 for $c=2.0455(\beta \approx 0.1899)$ and for $c=7.558(\beta \approx 0.21508)$ where the latter corresponds to the larger value of $W(0)$. In this figure, we also show the full numerical solution to (5.4b) with solid lines. We observe that the bump solution is essentially indistinguishable from the full numerical result even for the only moderately large value $c=7.558$ where $\beta$ lies close to $\beta_{1}=\frac{1}{\sqrt{2} \pi} \approx 0.225079$. Even for $c=2.0455$, the bump solution still provides a decent approximation of the true solution for small $y$, but differs from the numerical solution in the far field. We expect that the correspondence near the origin will improve by adding the higher order terms $W_{1}$ for the solution (see expression (5.7)). We remark that the value $\beta \approx 0.21508$ for $c=7.558$ corresponds to the terminal value of $\beta$ used in the slow sweep as shown in the right panel of Figure 7, and hence, lies close to $\beta_{1}$. 
Next, we will complete the asymptotic analysis and use a WKBJ-analysis to analyse (5.4b) for $|y| \gg \mathcal{O}\left(c^{-1 / 2}\right)$. By symmetry, we only need to consider the region on the right-hand side $y>0$ of the maximum of $W$. For this range of $y$, we assume that $W \ll 1$ and we can neglect the $W^{3}$-term in $(5.4 b)$ to obtain the linearized problem

$$
W_{y y}-\frac{y}{2} W_{y}-\left(c+\frac{1}{2}\right) W=0 .
$$

Upon introducing the Liouville transformation

$$
W=\exp \left(y^{2} / 8\right) U(y)
$$

we obtain that $U(y)$ satisfies

$$
U_{y y}-\left[c+\frac{1}{4}+\frac{y^{2}}{16}\right] U=0 .
$$

Applying the WKBJ-method then gives

$$
U=\frac{1}{\left[q_{0}(y)\right]^{1 / 4}}\left[a_{0} e^{\int^{y} \sqrt{q_{0}(\eta)} d \eta}+b_{0} e^{-\int^{y} \sqrt{q_{0}(\eta)} d \eta}\right], \quad \text { where } q_{0}(y) \equiv c+\frac{1}{4}+\frac{y^{2}}{16} .
$$

Next, we match this solution to the solution (5.12) in the bump region. We do this matching in the region where $c^{-1 / 2} \ll y \ll 1$ so that both solutions are valid. Then, the bump solution becomes to leading order

$$
W=2 \sqrt{2 c} e^{-\sqrt{c} y} .
$$

Since $y \ll 1(\ll \sqrt{c})$, the leading order of the integral in (5.16) can be determined, and using $W=U e^{y^{2} / 8}$ the WKBJ-solution becomes

$$
W(y)=c^{-\frac{1}{4}}\left[a_{0} e^{\sqrt{c} y}+b_{0} e^{-\sqrt{c} y}\right] .
$$

This can be matched directly to the bump solution by choosing $a_{0}=0$ and $b_{0}=2 \sqrt{2} c^{\frac{3}{4}}$. Note that this expression can be used provided that $y \ll \sqrt{c}$.

Using expression (5.16), we can also determine the behaviour of the solution in the far field where $|y| \gg \sqrt{c}$. Then, the solution becomes

$$
\begin{aligned}
W & =b_{0}\left(c+\frac{y^{2}}{16}\right)^{-1 / 4}\left[\frac{y}{2}+\sqrt{4 c+\frac{y^{2}}{4}}\right]^{-2 c-\frac{1}{2}} \exp \left(\frac{y^{2}}{8}-\frac{y}{2} \sqrt{c+\frac{1}{4}+\frac{y^{2}}{16}}\right) \\
& =2 b_{0} y^{-2 c-1} e^{-c-\frac{1}{4}}=4 \sqrt{2} c^{\frac{3}{4}} y^{-2 c-1} e^{-c-\frac{1}{4}}, \quad y \gg \mathcal{O}(\sqrt{c}),
\end{aligned}
$$

to leading order. This results in algebraic decay for $W$ in the far field. Hence, this WKBJanalysis has shown how the exponential decay of the bump solution makes a transition to algebraic decay in the far field.

Finally, we observe from (5.17) that, to leading-order, we can use the far-field behaviour $W \sim 2 \sqrt{2 c} e^{-\sqrt{c} y}$ of the bump solution on the entire range $\mathcal{O}\left(c^{-1 / 2}\right) \ll y \ll \mathcal{O}\left(c^{1 / 2}\right)$. 

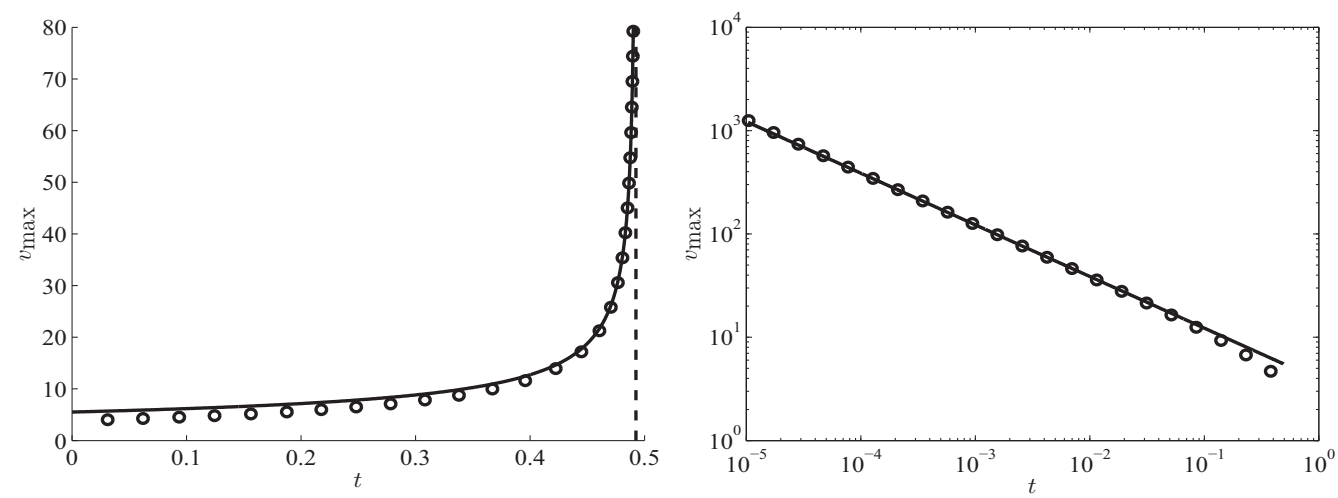

FIGURE 18. Left panel: Comparison of the asymptotic estimate for $v_{\max }$ given by $(5.19 \mathrm{~b})$ with that obtained from numerically solving the full nonlocal problem (1.3) (open circles) using FlexPDE6 [12]. The initial condition used in solving (1.3) is given by (5.19a) with $t=0$ and $T=1$. The parameters are $\varepsilon=0.01$ and $\beta=\beta_{1}-0.01$. Right panel: The blow-up time $T \approx 0.49255$ (vertical dashed line) in $(5.19 b)$ was estimated so that the numerical data appeared linear on the $\log -\log$ scale. In this panel, the corresponding log-log plot is given.

Therefore, the integral $\int_{-\infty}^{\infty} W^{3} d y$ can be evaluated asymptotically up to negligible exponentially small terms in $c$ by using only the approximate solution in the bump region. This justifies the calculation in (5.10)-(5.11).

In summary, upon returning to the dynamical rescaling (5.1), it follows that for $t \rightarrow T^{-}$ and for $\beta \rightarrow \beta_{1}^{-}$, where $\beta_{1} \equiv 1 /(\sqrt{2} \pi)$, a solution to (1.3) that blows up at $x=x_{0}$ is given by

$$
v \sim \frac{1}{\sqrt{T-t}} W\left[\frac{x-x_{0}}{\varepsilon \sqrt{T-t}}\right], \quad \text { where } W(y)=\sqrt{\frac{2}{3\left(1-\beta / \beta_{1}\right)}} \operatorname{sech}\left(y \sqrt{\frac{1}{3\left(1-\beta / \beta_{1}\right)}}\right),
$$

when $|y| \ll 1$. Therefore, we find that the maximum of the profile has the scaling behaviour

$$
v_{\max } \sim \frac{1}{\sqrt{T-t}} \sqrt{\frac{2}{3\left(1-\beta / \beta_{1}\right)}}, \quad \beta_{1} \equiv \frac{1}{\sqrt{2} \pi} .
$$

In Figure 18, we compare (5.19b) to the full numerical results in which the PDE (1.3) is solved using FlexPDE6 [12] when $\beta=\beta_{1}-0.01$ and $\varepsilon=0.01$. As initial condition, we use (5.19a) with $t=0$ and $T=1$. The numerical results are given by open circles. In the left panel of Figure 18, we plot $v_{\max }$ and see that these numerics correspond very well to the asymptotic expression (5.19b) for $v_{\max }$. From this plot, the blow-up time $T$ was estimated as $T \approx 0.49255$, given by the vertical dashed line. Using this, the plot can also be given on a $\log -\log$ scale as is done in the right panel of Figure 18. Note the very good agreement between the two results, solid and circles, even further away from the blow-up time.

Remark 5.1 The analysis above can be adjusted and applied to similar types of systems. For example, for the shadow limit resulting from the RD system obtained by replacing $\beta v^{3}$ by $\beta v^{2}$ 
in (1.1b) solutions that blow-up in finite time can also be shown to exist in an analogous way. This modified RD system was considered numerically in Section 5 of [19], where a blow-up behaviour of localized spike solutions was conjectured.

\subsection{Linear stability of the blow-up profile}

In this subsection, we study the linear stability of the blow-up profile. We do this in the context of the rescaled problem (5.2) and not for the original PDE (1.3). We substitute

$$
w(s, y)=W(y)+e^{\lambda s} \phi(y),
$$

into (5.2) where $\phi \ll 1$ and $W$ is the solution we constructed in the previous section. Upon neglecting the $e^{-s} w$ term, which vanishes when $t \rightarrow T^{-}$, we obtain the linearized problem

$$
\phi_{y y}-\frac{1}{2}(y \phi)_{y}-\beta \phi \int_{-\infty}^{\infty} W^{3} d y-3 \beta W \int_{-\infty}^{\infty} W^{2} \phi d y+3 W^{2} \phi=\lambda \phi .
$$

Then, after using the expression for $\beta$ in (5.4a), equation (5.21) results in the NLEP

$$
\phi_{y y}-\frac{1}{2}(y \phi)_{y}-c \phi-3 c W \frac{\int_{-\infty}^{\infty} W^{2} \phi d y}{\int_{-\infty}^{\infty} W^{3} d y}+3 W^{2} \phi=\lambda \phi
$$

where $W(y)$ is a solution of (5.4). In the construction of the solution $W$, we introduced the rescaling (5.5) and it is convenient to change to the same variables in equation (5.22). Therefore, we introduce the variables

$$
z=\sqrt{c} y, \quad \mathcal{W}(z)=c^{-1 / 2} W(z / \sqrt{c}), \quad \Phi(z)=\phi(z / \sqrt{c}),
$$

so that (5.22) transforms to

$$
\mathcal{L} \Phi \equiv L_{0} \Phi-3 \mathcal{W} \frac{\int_{-\infty}^{\infty} \mathcal{W}^{2} \Phi d z}{\int_{-\infty}^{\infty} \mathcal{W}^{3} d z}=\lambda_{0} \Phi+\frac{1}{2 c}(z \Phi)_{z}, \quad \text { where } \quad L_{0} \Phi \equiv \Phi_{z z}-\Phi+3 \mathcal{W}^{2} \Phi,
$$

and $\lambda_{0}=\frac{\lambda}{c}$. In (5.24), the blow-up profile $\mathcal{W}(z)$ satisfies equation (5.6), which is given by

$$
\mathcal{W}_{z z}-\mathcal{W}+\mathcal{W}^{3}=\frac{1}{2 c}\left(\mathcal{W}+z \mathcal{W}_{z}\right)
$$

We now show, as observed for other blow-up problems in [2], that (5.24) has two positive, but irrelevant, eigenvalues associated with shifts in the blow-up location and time.

Our result is as follows:

Lemma 5.1 The NLEP (5.24) has two positive eigenvalues associated with the nearsimilarity group variables (5.1). For all $c>0$, we have that $\Phi=\mathcal{W}_{z}$ and $\lambda_{0}=\frac{1}{2 c}$ is an eigenpair associated with translation of the blow-up location. Moreover, $\Phi=\mathcal{W}+z \mathcal{W}_{z}$ and $\lambda_{0}=\frac{1}{c}$ is an eigenpair associated with translation of the blow-up time. 
Proof To show that $\Phi=\mathcal{W}_{z}$ is a solution of (5.24), we substitute it into this equation. First, we determine $\mathcal{L} \mathcal{W}_{z}=\frac{1}{2 c}\left(\mathcal{W}_{z}+z \mathcal{W}_{z z}\right)+\lambda_{0} \mathcal{W}_{z}$. Next, we find that

$$
\mathcal{L} \mathcal{W}_{z}=L_{0} \mathcal{W}_{z}-3 \mathcal{W} \frac{\int_{-\infty}^{\infty} \mathcal{W}^{2} \mathcal{W}_{z} d z}{\int_{-\infty}^{\infty} \mathcal{W}^{3} d z}=\mathcal{W}_{z z z}-\mathcal{W}_{z}+3 \mathcal{W}^{2} \mathcal{W}_{z}=\frac{1}{2 c}\left(2 \mathcal{W}_{z}+z \mathcal{W}_{z z}\right)
$$

where we use that the integral $\int_{-\infty}^{\infty} \mathcal{W}^{2} \mathcal{W}_{z} d z$ is zero from the fact that $W \rightarrow 0$ as $z \rightarrow \pm \infty$. To obtain the last equality, we differentiate (5.25).

Upon comparing these two results, we conclude that $\lambda_{0}=\frac{1}{2 c}$. Therefore, $\Phi=\mathcal{W}_{z}$ and $\lambda_{0}=\frac{1}{2 c}$ is an eigenpair.

Now, we show that $\Phi=\mathcal{W}+z \mathcal{W}_{z}$ and $\lambda_{0}=\frac{1}{c}$ is an eigenpair. We first determine $\mathcal{L W}$ as

$$
\begin{aligned}
\mathcal{L} \mathcal{W} & =\left(\mathcal{W}_{z z}-\mathcal{W}\right)+3 \mathcal{W}^{3}-3 \mathcal{W}=\left(-\mathcal{W}^{3}+\frac{1}{2 c}\left(\mathcal{W}+z \mathcal{W}_{z}\right)\right)+3 \mathcal{W}^{3}-3 \mathcal{W}, \\
& =2 \mathcal{W}^{3}-3 \mathcal{W}+\frac{1}{2 c}\left(\mathcal{W}+z \mathcal{W}_{z}\right),
\end{aligned}
$$

where in the second equality we use the equation (5.25) for the blow-up profile. Then, we obtain $\mathcal{L}\left(z \mathcal{W}_{z}\right)$, again using the differentiated version of equation (5.25), as

$$
\begin{aligned}
\mathcal{L}\left(z \mathcal{W}_{z}\right) & =\left(z \mathcal{W}_{z}\right)_{z z}-z \mathcal{W}_{z}+3 \mathcal{W}^{2} \mathcal{W}_{z} z-3 \mathcal{W} \frac{\int_{-\infty}^{\infty} \mathcal{W}^{2}\left(z \mathcal{W}_{z}\right) d z}{\int_{-\infty}^{\infty} \mathcal{W}^{3} d z} \\
& =z\left(\mathcal{W}_{z z z}-\mathcal{W}_{z}+3 \mathcal{W}^{2} \mathcal{W}_{z}\right)+2 \mathcal{W}_{z z}-\mathcal{W} \frac{\int_{-\infty}^{\infty} z\left(\mathcal{W}^{3}\right)_{z} d z}{\int_{-\infty}^{\infty} \mathcal{W}^{3} d z} \\
& =\frac{z}{2 c}\left(2 \mathcal{W}_{z}+z \mathcal{W}_{z z}\right)+2 \mathcal{W}_{z z}+\mathcal{W}
\end{aligned}
$$

Upon adding (5.27) and (5.28), we find that

$$
\begin{aligned}
\mathcal{L}\left(\mathcal{W}+z \mathcal{W}_{z}\right) & =2\left(\mathcal{W}_{z z}-\mathcal{W}+\mathcal{W}^{3}\right)+\frac{1}{2 c}\left(\mathcal{W}+z \mathcal{W}_{z}\right)+\frac{z}{2 c}\left(2 \mathcal{W}_{z}+z \mathcal{W}_{z z}\right), \\
& =\frac{1}{c}\left(\mathcal{W}+z \mathcal{W}_{z}\right)+\frac{1}{2 c}\left(\mathcal{W}+z \mathcal{W}_{z}\right)+\frac{z}{2 c}\left(2 \mathcal{W}_{z}+z \mathcal{W}_{z z}\right),
\end{aligned}
$$

where we again use (5.25).

On the other hand, from the right-hand side of (5.24), we require that

$$
\mathcal{L}\left(\mathcal{W}+z \mathcal{W}_{z}\right)=\lambda_{0}\left(\mathcal{W}+z \mathcal{W}_{z}\right)+\frac{1}{2 c}\left(\mathcal{W}+z \mathcal{W}_{z}\right)+\frac{z}{2 c}\left(2 \mathcal{W}_{z}+z \mathcal{W}_{z z}\right)
$$

This expression agrees with (5.29) when $\lambda_{0}=\frac{1}{c}$. Therefore, $\Phi=\mathcal{W}+z \mathcal{W}_{z}$ and $\lambda_{0}=\frac{1}{c}$ is an eigenpair of (5.24).

As discussed in [2], these two positive eigenvalues arise as a consequence of the invariances with respect to translations in $x$ and $t$ of the original PDE, and the form of the variables of the dynamical rescaling (5.1). In equation (1.3), when $x_{0}$ is subject to a shift $x_{0} \rightarrow x_{0}+\delta$, one solution is transformed into another. In terms of the dynamical 
rescaling, this translated solution takes the form

$$
v(x, t)=\frac{1}{\sqrt{(T-t)}} W\left(\frac{x-\left(x_{0}+\delta\right)}{\varepsilon \sqrt{(T-t)}}\right)=e^{\frac{s}{2}}\left(W(y)-\delta \varepsilon e^{\frac{s}{2}} W_{y}(y)\right),
$$

where $\delta$ is small. Hence, the eigenvalue $\lambda$, as given in (5.20) is $\frac{1}{2}$ with eigenfunction $W_{y}$ so that after transforming with $c$, we get that $\lambda_{0}=\frac{\lambda}{c}=\frac{1}{2 c}$ is the eigenvalue with eigenfunction $\mathcal{W}_{y}$.

In a similar way, by perturbing the blow-up time $T$ to $T=T+\delta, 0<\delta \ll 1$, we find that the translated solution reads to leading order as

$$
\begin{aligned}
v(x, t) & =\frac{1}{\sqrt{(T+\delta-t)}} W\left(\frac{x-x_{0}}{\varepsilon \sqrt{(T+\delta-t)}}\right)=e^{\frac{s}{2}}\left(1-\frac{1}{2} \delta e^{s}\right) W\left(y\left(1-\frac{1}{2} \delta e^{s}\right)\right), \\
& =e^{\frac{s}{2}}\left(1-\frac{1}{2} \delta e^{s}\right)\left(W(y)-\frac{1}{2} \delta e^{s} W_{y}(y)\right), \\
& =e^{\frac{s}{2}}\left(W(y)-\frac{1}{2} \delta e^{s}\left[W+W_{y}(y)\right]\right),
\end{aligned}
$$

and hence, the eigenvalue $\lambda=1$ and $\lambda_{0}=\frac{\lambda}{c}=\frac{1}{c}$ is the eigenvalue with associated eigenfunction $\mathcal{W}+z \mathcal{W}_{z}$.

Fortunately, these positive eigenvalues do not lead to instability since our aim is to study the stability of one particular blow-up solution. Therefore, we do not allow for perturbations tangent to a family of blow-up solutions. However, the symmetries leading to the eigenvalues in Lemma 5.1, shifting $x_{0}$ and translating $T$ is a perturbation in the direction of a different blow-up solution. Hence, both of these eigenvalues do not need to be taken into account in a conclusion regarding the spectral stability of the solution.

Next, to determine whether (5.24) has other positive eigenvalues that would lead to a genuine instability. For this, we numerically compute the spectrum of $(5.24)$ in $\operatorname{Re}(\lambda)>0$. To do so, we discretize (5.24) to $\mathcal{O}\left(h^{2}\right)$ using centred differences for $\Phi_{z z}$ and $\left[z \Phi_{z}\right]_{z}$ on a uniform mesh with meshsize $h$, while using the trapezoidal rule for the non-local term. The eigenvalues of the resulting matrix problem are then computed numerically to determine the eigenvalues with the largest real parts. These computations used 400 uniformly spaced meshpoints on a domain $0<z<15$. For the case where $\Phi$ is even in $y$, we plot in Figure 19 the only positive eigenvalue of (5.24). This agrees to several significant digits with the positive eigenvalue $\lambda_{0}=\frac{1}{c}$ associated with a shift in the blow-up time $T$. Note that the corresponding eigenfunction of this eigenvalue is indeed even. A similar computation for the class of eigenfunctions for which $\Phi$ is odd reveals that $\lambda_{0}=\frac{1}{2 c}$ (which has an eigenfunction that is odd) is the only unstable eigenvalue (not shown). As a result, this numerical evidence shows that the blow-up profile is indeed stable.

Although it appears to be difficult to prove rigorously for all $c>0$ that (5.24) has no other positive eigenvalues other than the two associated with the near-similarity group, such a proof is easily given for the limiting problem where $c \rightarrow \infty$, corresponding to $\beta \rightarrow \beta_{1}$. In the limit $c \rightarrow \infty$, we have $\mathcal{W} \sim \mathcal{W}_{0}=\sqrt{2} \operatorname{sech} z$ and also the two positive eigenvalues associated with the similarity variables tend to zero. In addition, the NLEP 


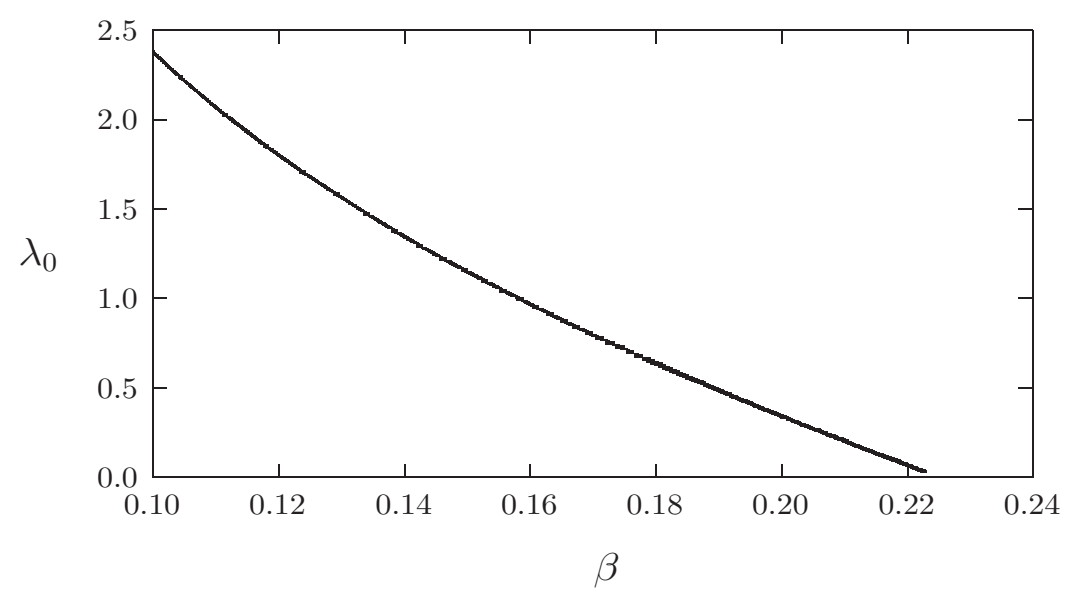

FIGURE 19. The only positive eigenvalue of (5.24) for the class of even eigenfunctions, as computed by discretizing (5.24) and computing the eigenvalues of a matrix eigenvalue problem. This only positive eigenvalue is, to within several digits of accuracy, the eigenvalue $\lambda_{0}=1 / c$ associated with a translation in the blow-up time.

(5.24) reduces to leading order to

$$
L_{0} \Phi-3 \mathcal{W}_{0} \frac{\int_{-\infty}^{\infty} \mathcal{W}_{0}^{2} \Phi d z}{\int_{-\infty}^{\infty} \mathcal{W}_{0}^{3} d z}=\lambda_{0} \Phi \quad \text { where } \quad L_{0} \Phi \equiv \Phi_{z z}-\Phi+3 \mathcal{W}_{0}^{2} \Phi
$$

From the proof of Lemma 5.1, it follows that $\lambda_{0}=0$ is an eigenvalue of multiplicity two, with eigenfunctions $\Phi=\mathcal{W}_{z}$ and $\Phi=\mathcal{W}+z \mathcal{W}_{z}$.

To show there are no positive eigenvalues for the limiting problem (5.30), we observe that (5.30) is an explicitly solvable NLEP in the sense of [25].

As such, following the proof of Lemma 3.1 above, we use Green's identity on $\mathcal{W}_{0}^{2}$ and $\Phi$ to obtain the usual integral identity $\int_{-\infty}^{\infty}\left(\mathcal{W}_{0}^{2} L_{0} \Phi-\Phi L_{0} \mathcal{W}_{0}^{2}\right) d z=0$. Upon using (5.30) for $L_{0}$, together with $L_{0} \mathcal{W}_{0}^{2}=3 \mathcal{W}_{0}^{2}$ (see Lemma 2.3 of [25]), this identity yields

$$
0=\left(3 \frac{\int_{-\infty}^{\infty} \mathcal{W}_{0}^{3} d z}{\int_{-\infty}^{\infty} \mathcal{W}_{0}^{3} d z}+\lambda_{0}-3\right) \int_{-\infty}^{\infty} \mathcal{W}_{0}^{2} \Phi d z=\lambda_{0} \int_{-\infty}^{\infty} \mathcal{W}_{0}^{2} \Phi d z
$$

Therefore, for eigenfunctions for which $\int_{-\infty}^{\infty} \mathcal{W}_{0}^{2} \Phi d z \neq 0$, we obtain that $\lambda_{0}=0$ is the only discrete eigenvalue. For eigenfunctions for which $\int_{-\infty}^{\infty} \mathcal{W}_{0}^{2} \Phi d z=0$, (5.30) yields the local eigenvalue problem $L_{0} \Phi=v \Phi$. It was proved in Proposition 5.6 of [6] that the point spectrum of this local eigenvalue problem consists only of $v_{0}=3$, for which $\Phi>0$ and consequently $\int_{-\infty}^{\infty} \mathcal{W}_{0}^{2} \Phi d z \neq 0$, together with translation mode $v_{1}=0$, associated with the odd eigenfunction $\Phi=\mathcal{W}_{0 z}$. As such, we conclude that the limiting problem (5.30) has no positive eigenvalue. 


\section{Discussion}

We have used a combination of asymptotic analysis, linear stability theory, and full numerical simulations to investigate intricate dynamical behaviour of spike-type solutions to a RD system that finally leads to finite-time blowup behaviour of a localized solution. By slowly ramping a bifurcation parameter linearly in time through various linear stability and existence thresholds associated with multi-spike steady-states, we show that there can be a dynamically intricate route, or transition, of either spike nucleation, spike annihilation, or temporal oscillation of the spike amplitude that precedes an ultimate finite-time blowup of a spike amplitude. Our analysis of this new type of spike behaviour was motivated by some numerical observations in [7] and [19].

From a mathematical viewpoint, our analysis, together with that in [33], provides one of the first detailed analyses of delayed bifurcation behaviour for localized structures in PDEs. Our study hints at the rather wide variety of routes to finite-time blow-up in PDE RD systems, as opposed to that which occurs in standard well-studied scalar models, such as those associated with quasilinear heat equations.

A key open problem is to provide a theoretical global existence analysis of solutions to (1.1). For the non-local GL model (1.3), we have used a combination of asymptotic analysis and numerical methods to suggest that finite-time blow-up at $x=0$ will occur when $\beta$ is below the existence threshold $\beta_{1}$ of a one-spike steady-state solution. To provide a rigorous proof of this conjecture would complete the picture. In addition, for those parameter ranges for the full RD system (1.1) where finite-time blow-up is observed, it would be interesting to extend the centre-manifold approach of [11] to provide a detailed characterization of the blow-up profile through the use of a near-similarity group transformation. In this direction, to analytically characterize the novel route to blow-up observed numerically in Figure 12, whereby the spike amplitude develops increasingly large oscillations, before apparently bouncing off to infinity, would be a challenge.

\section{Acknowledgements}

M. J. Ward was supported by an NSERC Discovery Grant 81541 (Canada). J. C. Tzou was supported by a PIMS postdoctoral fellowship.

\section{References}

[1] Baer, S. M., Erneux, T. \& Rinzel, J. (1989) The slow passage through a Hopf bifurcation: Delay, memory effects, and resonance. SIAM J. Appl. Math. 49(1), 55-71.

[2] Bernoff, A. J. \& Witelski, T. P. (2010) Stability and dynamics of self-similarity in evolution equations. J. Eng. Math. 66(1), 11-31.

[3] Budd, C., Dold, B. \& Stuart, A. (1993) Blowup in a partial differential equation with conserved first integral. SIAM J. Appl. Math. 53(3), 718-742.

[4] Budd, C., RottschäFer, V. \& Williams, J. F. (2008) Multi-bump, blow-up, self-similar solutions of the complex Ginzburg-Landau equation. Physica D 237(4), 510-539.

[5] Doelman, A., Gardner, R. A. \& Kaper, T. J. (1998) Stability analysis of singular patterns in the 1D Gray-Scott model: A matched asymptotics approach. Physica D 122, 1-36.

[6] Doelman, A., Gardner, R. A. \& Kaper, T. J. (2001) Large stable pulse solutions in reactiondiffusion equations. Indiana U. Math. J. 50(1), 443-507.

[7] Doelman, A. \& Kaper, T. (2003) Semistrong pulse interactions in a class of coupled reactiondiffusion equations. SIAM J. Appl. Dyn. Sys. 2(1), 53-96. 
[8] EI, S., Nishiura, Y. \& Ueda, K. (2001) $2^{n}$ splitting or edge splitting?: A manner of splitting in dissipative systems. Japan. J. Indust. Appl. Math. 18(2), 181-205.

[9] Erneux, T., Reiss, E. L., Holden, L. J. \& Georgiou, M. (1991) Slow passage through bifurcation and limit points. Asymptotic theory and applications. In: Dynamic Bifurcations (Luminy 1990), Lecture Notes in Math., Vol. 1493, Springer, Berlin, pp. 14-28.

[10] FilipPas, S. \& Guo, J. S. (1993) Quenching profiles for one-dimensional semilinear heat equations. Quart. Appl. Math. 51(4), 713-729.

[11] Filippas, S. \& KoHn, R. V. (1992) Refined asymptotics for the blow up of $u_{t}-\Delta u=u^{p}$. Comm. Pure Appl. Math. 45(7), 821-869.

[12] FlexPDE6, PDE Solutions Inc. URL: http://www.pdesolutions.com

[13] GaO, W. J. \& Han, Y. Z. (2011) Blow-up of a nonlocal semilinear parabolic equation with positive initial energy. Appl. Math. Lett. 24(5), 784-788.

[14] Gierer, A. \& Meinhardt, H. (1972) A theory of biological pattern formation. Kybernetik 12, 30-39.

[15] Iron, D., Ward, M. J. \& WeI, J. (2001) The stability of spike solutions to the one-dimensional Gierer-Meinhardt model. Physica D 150(1-2), 25-62.

[16] Iron, D. \& WARD, M. J. (2002) The dynamics of multi-spike solutions for the one-dimensional Gierer-Meinhardt model. SIAM J. Appl. Math. 62(6), 1924-1951.

[17] JAZAR, M. \& KiWAN, R. (2008) Blow-up of a non-local semilinear parabolic equation with Neumann boundary conditions. Ann. Inst. H. Poincar Anal. Non Linaire 25, 215-218.

[18] Kolokolnikov, T., Ward, M. \& WeI, J. (2005) The stability of spike equilibria in the one-dimensional Gray-Scott model: The pulse-splitting regime. Physica D 202(3-4), 258-293.

[19] Kolokolnikov, T., Ward, M. J. \& Wei, J. (2005) Pulse-splitting for some reaction-diffusion systems in one-space dimension. Stud. Appl. Math. 114(2), 115-165.

[20] Kolokonikov, T., Ward, M. J. \& WeI, J. (2014) The stability of hot-spot patterns for a reaction-diffusion system of Urban crime. DCDS-B 19(5), 1373-1410.

[21] Kuehn, C. (2011) A mathematical framework for critical transitions: Bifurcations, fast/slow systems and stochastic dynamics. Physica D 240(12), 1020-1035.

[22] Mandel, P. \& Erneux, T. (1987) The slow passage through a steady bifurcation: Delay and memory effects. J. Stat. Phys. 48(5-6), 1059-1070.

[23] Matthews, P. C. \& Cox, S. M. (2000) Pattern formation with a conservation law. Nonlinearity 13(4), 1293-1320.

[24] Moyles, I., Tse, W. H. \& Ward, M. J. (2016) On explicitly solvable nonlocal eigenvalue problems and the stability of localized stripes in reaction-diffusion systems. Stud. Appl. Math. 136(1), 89-136.

[25] Nec, Y. \& WARD, M. J. (2013) An explicitly solvable nonlocal eigenvalue problem and the stability of a spike for a sub-diffusive reaction-diffusion system. Math. Modeling Natur. Phen. 8(2), 55-87.

[26] Norbury, J., Wei, J. \& Winter, M. (2002) Existence and stability of singular patterns in a Ginzburg-Landau equation coupled with a mean field. Nonlinearity 15(6), 2077-2096.

[27] Nishiura, Y. \& Ueyama, D. (1999) A skeleton structure of self-replicating dynamics. Physica D 130(1-2), 73-104.

[28] Riecke, H. \& Rappel, W. J. (1995) Coexisting pulses in a model for binary-mixture convection. Phys. Rev. Lett. 75, 4035-4038.

[29] RotTsChäFer, V. (2013) Asymptotic analysis of a new type of multi-bump, self-similar, blowup solutions of the Ginzbrug Landau equation. Europ. J. Appl. Math. 24(91), 103-129.

[30] Soufi, E., Jazar, M. \& Monneau, R. (2007) A gamma-convergence argument for the blow-up of a nonlocal semilinear parabolic equation with Neumann boundary conditions. Ann. Inst. H. Poincaré-Anal. Non Lineaire 24, 17-39. 
[31] Souplet, P. (1999) Uniform blowup profiles and boundary behavior for diffusion equations with nonlocal nonlinear source. J. Diff. Eq. 153(2), 374-406.

[32] Souplet, P. (1998) Blowup in nonlocal reaction-diffusion equations. SIAM J. Math. Anal. 29(6), 1301-1334.

[33] Tzou, J. C., Ward, M. J. \& Kolokolnikov, T. (2015) Slowly varying control parameters, delayed bifurcations, and the stability of spikes in reaction-diffusion systems. Physica $D$ 290(1), 24-43.

[34] Wang, X., Tian, F. \& Li, G. (2015) Nonlocal parabolic equation with conserved spatial integral. Arch. Math. 105(1), 93-100.

[35] Ward, M. J. \& WeI, J. (2003) Hopf bifurcations and oscillatory instabilities of spike solutions for the one-dimensional Gierer-Meinhardt model. J. Nonlinear Sci. 13(2), 209-264.

[36] Wei, J. \& Winter, M. (2005) On a Cubic-Quintic Ginzburg-Landau equation with global coupling. Proc. Amer. Math. Soc. 133(6), 1787-1796. 